\title{
Mechanistic Analysis of Light-Driven Overcrowded Alkene-Based Molecular Motors by Multiscale Molecular Simulations
}

\author{
Mudong Feng and Michael K. Gilson
}

December 29, 2020

\begin{abstract}
Department of Chemistry and Biochemistry, and Skaggs School of Pharmacy and Pharmaceutical Sciences, UC San Diego, La Jolla, CA 92093. Email:mgilson@health.ucsd.edu
\end{abstract}

\begin{abstract}
We analyze light-driven overcrowded alkene-based molecular motors, an intriguing class of small molecules that have the potential to generate MHz-scale rotation rates. The full rotation process is simulated at multiple scales by combining quantum surface-hopping molecular dynamics (MD) simulations for the photoisomerization step with classical MD simulations for the thermal helix inversion step. A Markov state analysis resolves conformational substates, their interconversion kinetics, and their roles in the motor's rotation process. Furthermore, motor performance metrics, including rotation rate and maximal power output, are computed to validate computations against experimental measurements and to inform future designs. Lastly, we find that to correctly model these motors, the force field must be optimized by fitting selected parameters to reference quantum mechanical energy surfaces. Overall, our simulations yield encouraging agreement with experimental observables such as rotation rates, and provide mechanistic insights that may help future designs.
\end{abstract}

\section{Introduction}

Molecular motors are molecules that transduce optical or chemical energy into mechanical motions, such as translation and rotation, through cyclic conformational changes [1]. Naturally occurring molecular motors, such as ATP synthase and myosin, have evolved to support many essential biological functions [2].It has also been argued that even enzymes not usually thought of as molecular motors, such as adenosine kinase, must exhibit at least weak motor-like properties, as a direct consequence of their being thrown back and forth between multiple chiral free energy surfaces corresponding to their apo and substrate-bound states [3]. The same fundamental principles also apply to non-biological molecules, and indeed, exciting progress has also been made in the design and synthesis of artificial molecular motors that may mimic or even surpass their biological counterparts $[4,1,5]$.

In particular, the overcrowded alkene-based motors $[6,7]$ (abbreviated here as alkene motors) generate robust unidirectional rotation, which has been harnessed in several demonstration applications $[8,9,10,11]$. The alkene motors have a central double bond between two carbons (Fig 1), which divides the molecule into a lower part, referred to as the stator, and an upper part, referred to as the rotor. If one views the motor, in the stereochemistry shown, along the central bond, from the rotor to the stator, the rotor turns clockwise relative to the stator. In the first step, the central double bond undergoes Z-E photoisomerization, converting the stable form (A) to the metastable form (B). In the second step, thermal helix inversion (THI) converts B to another stable form (C). Here, the change in stator pucker from $\mathrm{B}$ to $\mathrm{C}$ resembles a flap of butterfly wings, putting the stator benzene rings from the back to the front. Another, similar, $180^{\circ}$ rotation then converts $\mathrm{C}$ to A via D. Because the motors shown here have symmetric stators, A and C have identical energy, as do B and D. In contrast, A and 

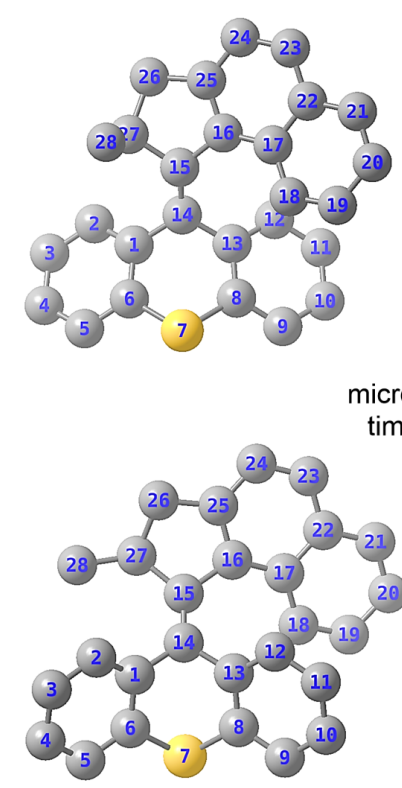

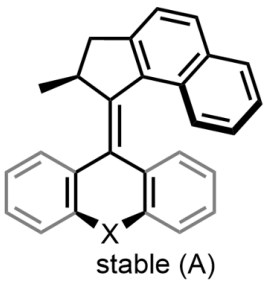

icrosecond
timescale $\begin{gathered}\text { room } \\ \text { temperature }\end{gathered}$

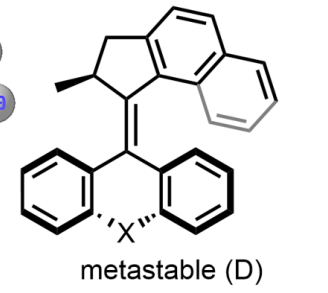

Motor $\mathrm{S}$ : $\mathrm{X}=\mathrm{S}$

Motor N: $X=\mathrm{N}-\mathrm{COOt}-\mathrm{Bu}$

Motor $\mathrm{O}$ : $\mathrm{X}=\mathrm{O}$ (stator is planar, unlike conformations drawn)
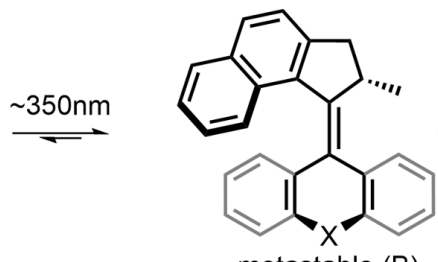

metastable (B)
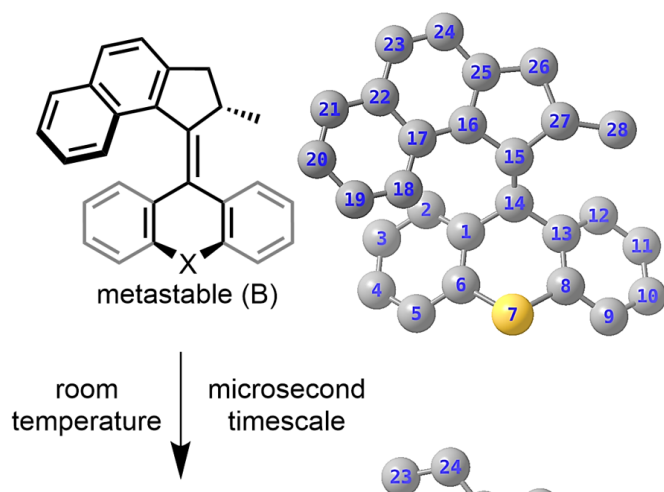

$\stackrel{\sim 350 \mathrm{~nm}}{\sim}$
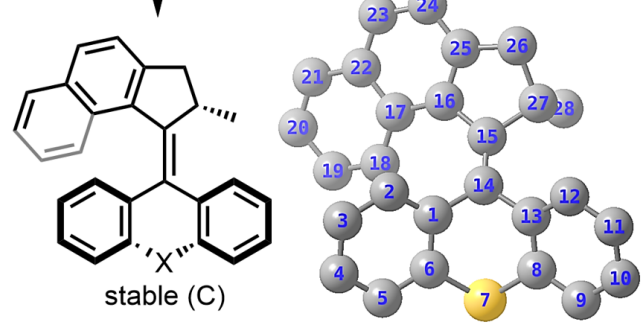

Figure 1: Chemical structures and rotation processes of the molecular motors studied in this paper. The rate constants for their THI steps (vertical arrows) all are on the microsecond timescale $\left(8 \times 10^{6} \mathrm{~s}^{-1}\right.$, $2 \times 10^{6} \mathrm{~s}^{-1}, 3 \times 10^{5} \mathrm{~s}^{-1}$ for Motors $\mathrm{S}, \mathrm{N}, \mathrm{O}$ respectively $\left.[16,12]\right)$. Although the metastable forms have multiple substates, only the lowest energy metastable substate is drawn here. The corresponding $3 \mathrm{D}$ structures provide atom numberings used throughout this study.

$\mathrm{D}$ always have different stabilities, as do $\mathrm{B}$ and $\mathrm{C}$, due to difference in ring pucker. Thus, the pucker of the rotor 5-member ring puts the methyl group in either axial $(\mathrm{A}, \mathrm{C})$ or equatorial $(\mathrm{B}, \mathrm{D})$ position, the latter giving larger repulsion with the stator, and hence higher energy [12]. As previously reported $[13,14,15]$, the metastable forms, $\mathrm{B}$ and $\mathrm{D}$, are actually mixtures of metastable substates with distinct conformations, and hence are more complex than shown in Fig 1.

An intuitive explanation of this process in terms of motions on the ground and excited state potential energy surfaces (PES) is provided in Fig 2 [16]. The motor starts in stable form (A) and is excited by light to a Franck-Condon (FC) point. It then rotates down the gradient of the excited state PES. During this critical step, it "flies over" a large ground state energy barrier that would be very difficult to pass on the ground state PES. When it is near the conical intersection between the excited state and ground state surfaces, the molecule hops back to its ground state and continues down the energy gradient to the metastable form (B). Because there is now a high ground state energy barrier blocking a reverse motion back to $\mathrm{A}$, the motor remains in $\mathrm{B}$ until thermal fluctuations kick it over the lower THI barrier to stable form (C). From C, the same process repeats, moving the motor through metastable form (D) and back to stable form (A).

These motors can be viewed as examples of flashing ratchets $[5,17]$, where switching between two different energy surfaces - here the ground state and the excited state- drives directional motion. In the absence of light, the motor would go to equilibrium on the ground state PES, and its conformational probabilities would follow the Boltzmann distribution. Consequently, the stable form would be at much higher concentration than the metastable form, and the principle of detailed balance [17] would mean that no net rotation occurs. In the presence of a constant light source, photo-excitations break detailed balance and create a non-Boltzmann steady-state distribution, the so-called photostationary state. Unlike in equilibrium, the steady state concentration of the metastable form can match or surpass the stable form $[18,16]$. Thus, the motor molecules can transduce light energy by letting photoisomerizations pump them to the metastable form, and releasing their stored energies during 


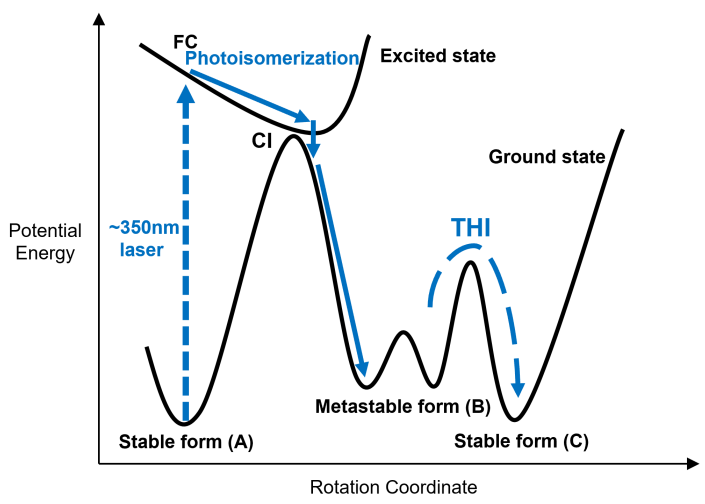

Figure 2: Motor rotation dynamics on the ground state (lower) and excited state (upper) potential energy surfaces. FC: Frank-Condon point. CI: conical intersection. THI: Thermal helix inversion. Stable and metastable forms are described in Figure 1).

THI.

Given the ultimate goal of using molecular motors for practical applications, it is of interest to consider performance metrics to be optimized when designing them. One obvious metric is the rotation speed. There have been considerable efforts to make faster alkene motors, as reviewed from both experimental [6,7] and theoretical perspectives [19]. These have focused on accelerating THI, which is the rate-determining step of the rotational cycle, without sacrificing unidirectionality. The THI rate constant can be measured experimentally by monitoring the relaxation of the absorption spectrum after pulse excitation [16]. It can also be obtained from molecular simulations by determining the mean first passage time for the transition from the metastable form to the stable form. Thus, comparing to experiments provide a way to gauge simulation accuracy. Another performance metric, the power output, equals the rotation speed times the average work the motor does in each rotation cycle. However, the power output depends on factors extrinsic to the motor, notably the magnitude of load and how the load is coupled to the motor. A more general and intrinsic property is the thermodynamic maximum of the work per cycle, which is related to the free energy released when the motor undergoes THI.

Important prior studies have developed quantitative models of these molecular motors at varying levels of molecular detail. Geertsema et al. constructed a Markov state model (MSM) with rate constants fitted to NMR measurements [18], and evaluated serveral performance metrics such as the average rotation speed and the degree of rotation unidirectionality. Their model provides a valuable perspective of concentration fluxes in the system at a macroscopic level, but does not offer microscopic details beyond the simple dichotomy of stable vs. metastable form. Other studies have considered the microscopic level. Thus, computational studies have used static quantum mechanical (QM) methods that can provide accurate energies $[14,20]$, but these are not fully informative about the statistical mechanics of the rotational process. Short molecular dynamics (MD) simulations at the picosecond timescale have also been used to examine the photoisomerization step but not the slower THI step $[21,22,23,24]$. However, classical MD can now routinely reach the microsecond timescale for such small systems, and thus is directly applicable to the subset of motors with THI rate constants on the microsecond time scale $[16,12,25]$. These motors are more challenging to characterize experimentally, because of their high rates, but they are easier to characterize with classical MD.

Here, we propose a multiscale modeling framework that couples QM surface-hopping MD, used to model the photoisomerization step, with classical MD, used to model the THI step (Fig 3). The accuracy of our classical MD simulations is supported by optimization of selected force field parameters to fit QM energies and gradients for these specific compounds. The rotation mechanism is elucidated by full rotation trajectories (orange arrows in Fig 3) and Markov state models that provide conformations and interconversion kinetics of the metastable substates. We also assess motor performance by using weighted ensemble simulations to compute the THI rate constant, and umbrella sampling to compute 


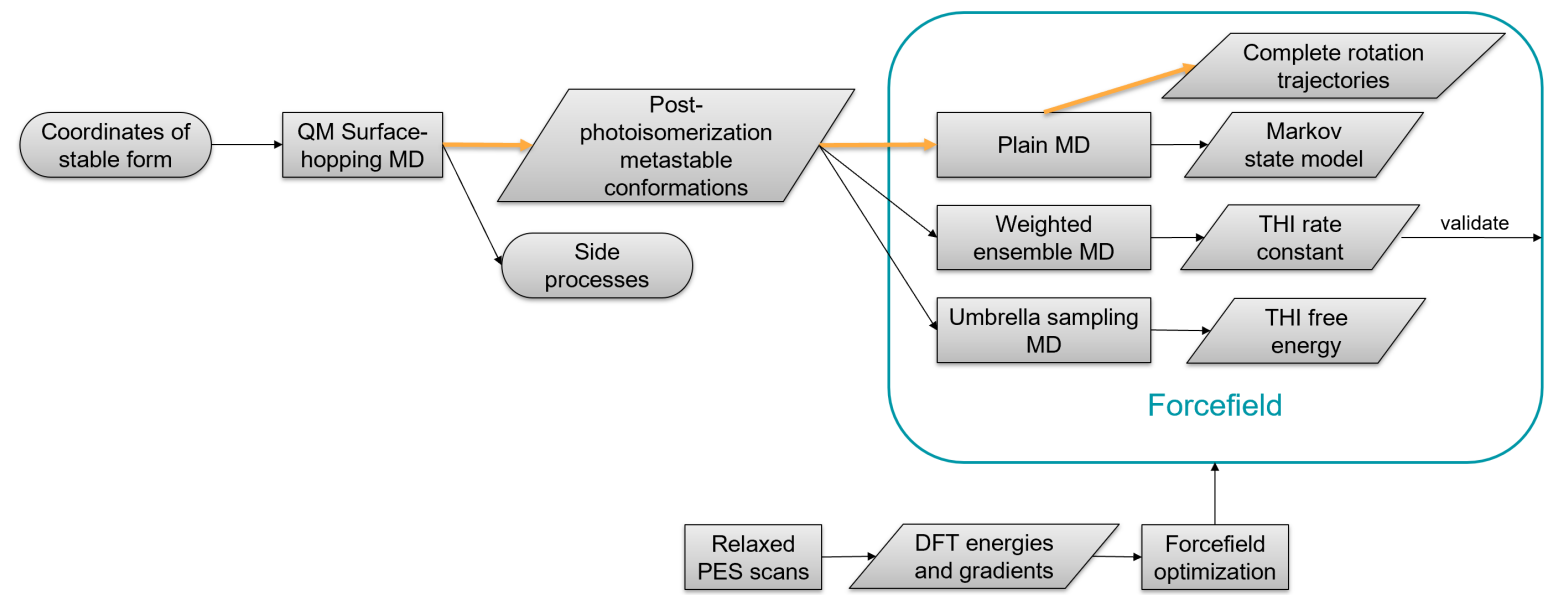

Figure 3: Workflow describing the proposed computational framework. Rectangles represent processes, whereas non-rectangular parallelograms stand for results/data. All force field-based methods and their results are encompassed by a rounded rectangle standing for the force field used. Combining the MD results along the orange arrows give complete $180^{\circ}$ rotation trajectories.

the free energy drop going from the metastable form to the stable form. Encouragingly, the THI rate constants computed agree well with experimental measurements. The results thus may benefit future design of similar molecular motors.

\section{Methods}

The computational framework in this study, illustrated in Fig 3, has three main components. First, the photoisomerization process is modeled with QM surface-hopping MD; second, classical MD at the microsecond timescale is used to study the THI mechanism and obtain quantities related to performance, such as rate constants and free energies. Finally, to achieve realistic classical MD results, we adjust force field parameters against conformational energies from reference QM calculations. The three components are described in the following subsections. Additional details are provided in the Electronic Supplementary Information (ESI); scripts and input files to reproduce this study can be downloaded from a Github repository (github.com/fengmudong/motor-paper).

\subsection{Surface-hopping MD simulation}

We used QM surface-hopping MD, run in replicates, to model the photoisomerization step of the motors in Fig 1. These simulation replicates provide a diverse and physically relevant sample of post-photoisomerization structures that serve as initial structures of our classical MD simulations.

Surface-hopping simulations were carried out at the OM2/MRCI level, which is a semi-empirical QM method designed for fast excited state calculations [26]. The software package MNDO [27] was used for these calculations. We first ran $10 \mathrm{ps}$ of sampling in the ground state, starting from the optimized structure of the stable form (3D structure C in Fig 1). Then, samples drawn at random from this initial ground state trajectory were set to the first excited state without any change in nuclear coordinates, if they met standard MNDO criteria such as successful mapping of the active space orbitals, and if they passed a stochastic selection based on the computed excitation probability [27]. About $70 \%$ of the initial samples passed and were excited. These excited samples represent an ensemble of Franck-Condon points, and from each we started a simulation replicate. Tully's fewest switches algorithm [28] was used to determine hopping to the ground state. Energies and gradients were evaluated on the fly at the OM2/MRCI level until the fixed simulation length of 4.5ps was reached. A replicate was kept for further use if it had hopped to the ground state and ended the 4.5ps run in the metastable form. The final coordinates of such replicates were used as initial coordinates of 
classical MD simulations. The other replicates, representing the side processes in Fig 3, were not used to constitute the full rotation trajectory, but offer additional insights, as discussed in the Results and Discussion section.

These surface-hopping simulations used a Langevin thermostat [29] targetting 90K, the temperature used in the relevant photoisomerization experiments [16], with a collision frequency of $0.2 / \mathrm{ps}$. The Langevin thermostat provides a random "kick" to each atom at every time-step, to model solvent impacts, and a drag on each atom, proportional to its velocity, to model the effect of solvent viscosity. The balance of these energy-adding and damping forces effectively sets the target simulation temperature. We also tried the Nose-Hoover thermostat[29], an alternative to the Langevin thermostat, but this led to non-physical low-frequency kinetic energy oscillations not present in Langevin or constant energy simulations, even when a long Nose-Hoover chain was used, so we chose not to use this method.

\subsection{Classical MD simulation}

First, classical MD simulations were appended to the QM surface-hopping MD trajectories (Section 2.1) to construct full rotation trajectories of Motor S and Motor N. This was not done for Motor O, for reasons detailed in Section 2.3.) The initial structure of each classical simulation was set as the final structure of one of the QM surface-hopping trajectories in the metastable form (Section 2.1). One motor molecule was solvated with 600 molecules of dichloromethane in a $40 \AA$ cubic periodic box, using the software Packmol [30]. After energy minimization, the solute was restrained in position while the system at constant volume was heated to $300 \mathrm{~K}$ with the Berendsen thermostat. It was then equilibrated, with restraints still on, at $300 \mathrm{~K}$ and a pressure of 1 bar with the Langevin thermostat and the Monte Carlo barostat, mimicking the experimental conditions for microsecond THI [16]. Finally, restraints were removed for $1 \mu$ s production runs at the same temperature and pressure. In classical $\mathrm{MD}$, the Langevin collision frequency we mainly use was $1 / \mathrm{ps}$, but different values were also used to evaluate the effect of this parameter on the kinetics (discussed later in Section 3.3.4). The trajectories that completed THI were concatenated with their corresponding surface-hopping MD trajectories to give continuous trajectories of the full $180^{\circ}$ motor rotation.

Then, these production MD trajectories (whether completed THI or not) were used to construct a reversible Markov state model (MSM) of each motor. The trajectory segments in the stable form after THI were excluded when building the MSM, because the reverse THI process was not sampled. The high dimensional trajectories of atomic positions were first condensed to time series of all the dihedrals present in the topology file of the motor; each dihedral is associated with a force field term. After further dimension-reduction using time-lagged independent component analysis (TICA), the time series in continuous conformational space was discretized to conformational clusters using k-means clustering. Then the clusters were classified by Perron cluster analysis (PCCA) [31] into 4 metastable substates. We chose 4 substates because partitioning the metastable form into a larger number of substates led to some substates being overly similar to each other. The time series of substate classification were then analyzed to produce the MSM, as a $4 \times 4$ transition matrix.

Although plain MD yielded a few trajectories that completed THI, many more such runs would be needed to obtain better precision for THI rate constants [32]. Therefore, we instead used an enhanced sampling technique, the weighted ensemble method implemented in WESTPA [33], to compute the THI rate constant. In WESTPA, a collection of MD replicates initiated from metastable form is managed by cloning new ones in regions of the conformational space with fewer replicates, and merging existing ones in regions with more replicates, to generate a more even distribution of replicates along desired progress coordinates. This allows an artificial increase in the number of replicates that sample higher energy transitional regions. Correct statistics is maintained by adjusting replicate weights when replicates are cloned or merged. Note that this method does not involve modifying the Hamiltonian. As detailed in Results and Discussion, THI progress coordinates were defined for each motor in terms of key structural features, such as dihedrals describing rotation around the central double bond and rotor/stator puckering coordinates. Then the conformational space was divided into bins of different progress coordinate values, and WESTPA was used to monitor the number of MD replicates in each bin to decide when to clone or merge replicates. When a replicate reached the bin defining the stable form of the motor, the replicate was restarted at one of the initial metastable conformations. As the 
probability distribution across bins converge to steady state distribution, the THI rate constant is calculated using the steady state flux from the metastable form to the stable form.

We calculated the free energy difference between the stable and metastable forms from potentials of mean force (PMF) obtained with umbrella sampling [34]. To do this, we used harmonic potentials of spring constant spring constant $200 \mathrm{~kJ} \mathrm{~mol}^{-1}$ to restrain the central double bond torsion angle in a series of $1 \mu \mathrm{s}$ MD simulation windows. The torsion value at each window center ranges from -1.9 to 2.1 radian, with 0.1 radian spacing. The central torsion angle is defined here as $43-15-14-29$, where 43 and 29 are hydrogens attached to $\mathrm{C} 18$ and C2 in Fig 1, respectively. This range spans the metastable form, the stable form, and the barrier between them. Umbrella sampling simulations were set up using the open-source library PLUMED [35, 36], and the PMF along the torsion was obtained by Weighted Histogram Analysis Method (WHAM) [37, 38]. From the PMF $F(x)$, which is essentially the conditional probability distribution expressed as a free energy profile, the free energy difference between the metastable form and the stable form was calculated by integrating across the corresponding ranges of the torsion values: $G_{m}-G_{s}=R T \ln \int_{s} e^{-\beta G(x)} d x-R T \ln \int_{m} e^{-\beta G(x)} d x$. Here the $\mathrm{s}$ and $\mathrm{m}$ subscripts indicate the sampling windows that span the stable and metastable forms, respectively.

\subsection{Optimization}

As detailed in Results and Discussion, we found that an initial "off the shelf" force field did not yield realistic results in classical MD simulations of these molecules. We therefore optimize selected force field parameters by fitting them to reference QM energy surfaces.

\subsubsection{Generation of QM potential energy surfaces}

Because the classical MD simulations address only ground state dynamics, ground state QM suffices to generate reliable fitting targets, including energies and energy gradients with respect to atomic positions. We chose DFTD3/DZVP level of theory, as it has been shown to strike a favorable balance between accuracy and computational cost in calculating conformational energies for molecules of this size [39]. The motor rotation process of interest involves conformations where the central carboncarbon bond is twisted, so that atoms 16-15-14-13 are not coplanar. In such conformations, $\pi$ orbital overlap is compromised, resulting in open shell character of the electronic structure; this motivated us to use unrestricted DFT orbitals.

We computed two dimensional PES by running constrained geometry optimizations at a grid of points along two predefined scan coordinates. Based on prior analyses of these motors $[13,24]$ and our own trial results, we chose two combinations of scan coordinates: central torsion angle 18-15-14-10 combined with pyramidalization 14-1-13-15, and central torsion angle 18-15-14-10 combined with rotor pucker 27-15-16-25.

We used the wavefront propagation algorithm implemented in TorsionDrive [40], an open source package, to generated QM surfaces of motors $\mathrm{S}, \mathrm{N}$ and $\mathrm{O}$ (Figure 1). TorsionDrive runs geometry optimizations constrained at each grid point multiple times, from different propagating directions, to find the lowest energy structure at that point. TorsionDrive also benefits from using multiple starting structures to seed the propagation; the seed structures we used were the final metastable form structures from QM surface-hopping MD, plus the structures shown in Fig 1. Relative to less exhaustive scanning methods in the literature, such strategy is less sensitive to convergence failures at some grid points, to hysteresis (i.e. different results when the same point is scanned from different direction), and to optimizations being trapped in local minima rather than the lowest energy structure at the grid point. As shown previously [40], these problems can be particularly severe for alkene motors, likely due to nontrivial size (48 atoms for Motor S) and high rotation barriers, but problems are largely alleviated by the TorsionDrive method. Energies and gradients were computed by the Psi 4 package [41] for use in TorsionDrive. 


\subsubsection{Parameter optimization}

We used a Newton-Raphson algorithm implemented in the open-source package ForceBalance [42] to systematically optimize force field parameters, so that the force field replicates as much as possible the QM energy surfaces (Section 2.3.1). During optimization, force field energies and gradients of the unsolvated motor molecule were compared with the corresponding QM energies and gradients. The objective function is the sum of squared energy and gradient differences between the force field and QM across the grid of conformations. For Motor $\mathrm{O}$, it proved necessary to employ additional regularization terms, i.e. penalty terms to discourage unphysically large changes of certain parameters, as described in Results and Discussion.

As detailed in Results and Discussion, we tested various choices of what parameters to optimize, and we found that optimizing dihedral (proper and improper) amplitudes alone sufficed for Motor $\mathrm{S}$ and $\mathrm{N}$, whereas Motor $\mathrm{O}$ needed more changes. Our procedures ensure that these parameters remain non-negative during optimization. No changes were considered to the functional form of the force field, the multiplicities and phases of torsional terms, and the partial atomic charges. Initial force field parameters were taken from general Amber force field (GAFF) version $2.1[43,44]$ implemented in AmberTools18 [45], with AM1/BCC partial charges [46]. A subtlety of atom type selection for the motors is discussed in the Appendix C. For the dichloromethane solvent at room temperature and pressure, the GAFF2 parameters gave a density of $1.25 \mathrm{~g} \mathrm{~cm}^{-3}$, in good agreement with the experimental value of $1.33 \mathrm{~g} \mathrm{~cm}^{-3}$ [47], so the GAFF2 parameters were used as is for the solvent.

\subsection{Selection of reaction coordinates}

It is often necessary to map chemically meaningful conformational differences to a few coordinates, which may be followed over time and through transitions. Here, we call these reaction coordinates, but other synonyms are often used; e.g., progress coordinates or collective variables. In general, intuition based on one coordinate alone, such as the central torsion angle, can be unreliable; it is preferable to account for all degrees of freedom in the system using methods like MD simulations, and then verify hypotheses about key coordinates through analysis of the resulting trajectories. For the motors in this study, the key conformational features are the rotation of the rotor relative to the stator, the pucker of the stator, the pucker of the rotor, and the pyramidalization of the two central carbons. We found that one well-chosen reaction coordinate suffices to differentiate between the metastable form and the stable form, as in the umbrella sampling calculations; however, at least two coordinates are needed to differentiate all the conformationally distinct and kinetically separated metastable substates. The approach used to define reaction coordinates in this study is detailed in the Appendix B.

\section{Results and Discussion}

\subsection{Modeling photoisomerization with QM surface-hopping MD}

\subsubsection{Analysis and fate of the simulation trajectories}

For each of the three motors in Fig 1, the photoisomerization step was simulated in multiple replicates that represent an ensemble of Franck-Condon points (Section 2.1). As summarized in Table 1, about $10 \%$ of the simulation replicates of Motor S and Motor O progressed to the ground state metastable form. Each of these simulations offers a chemically reasonable realization of photoisomerization. The trajectories are similar to those of other alkene motors in prior works $[22,24]$, in the sense that the conformations at which the excited to ground state hops occur are similar, as are the rotational processes. A video of a representative trajectory of Motor S is provided in the ESI (mndo-amber.mp4). Final ground state metastable form conformations of Motor S and Motor O were used as starting conformations for the classical MD simulations detailed in Section 3.3. The starting conformations excluded a few that had not yet relaxed to a sufficiently low-energy conformation and thus were not suitable starting points for the classical simulations. This left 13 and 4 initial conformations for Motors $\mathrm{S}$ and $\mathrm{O}$, respectively. For Motor N, the QM simulations did not yield any hops from the excited state to the ground state, despite running for similar durations and numbers of replicates. 
Table 1: Outcomes of surface-hopping molecular dynamics simulation replicates. Columns, from left to right, provide the motor name, the duration of each individual replicate simulation for this motor, the number of simulation replicates, the total number of replicates that failed to isomerize away from the initial stable form, the number of replicates remaining in the excited state, the number that completed photoisomerization and terminated in the metastable form, and the number that completed both photoisomerization and THI and thus terminated in the stable form. (All replicates that completed photoisomerization also hopped from the excited state to the ground state.)

\begin{tabular}{lllllll}
\hline \multirow{2}{*}{ Motor } & \multirow{2}{*}{ Time per replicate $(\mathrm{ps})$} & \multirow{2}{*}{ Replicates } & \multicolumn{3}{c}{ Not Isomerized } & \multicolumn{2}{c}{ Isomerized } \\
\cline { 4 - 7 } & & & Total & Still in excited state & Metastable Form & THI Completed \\
$\mathrm{S}$ & 4.5 & 194 & 170 & 120 & 21 & 3 \\
$\mathrm{O}$ & 4.5 & 71 & 64 & 55 & 6 & 1 \\
$\mathrm{~N}$ & 5.0 & 71 & 71 & 71 & 0 & 0 \\
\hline
\end{tabular}

In an interesting side-process, a few replicates of Motors $\mathrm{S}$ and $\mathrm{O}$ actually completed THI (Table 1), progressing through the metastable form to the stable form. In all three of these simulations for Motor $\mathrm{S}$, the transition to the stable form is immediately preceded by flipping of the stator pucker to that of the target stable form, as seen in the representative trajectory (video mndo-whole.mp4 in the ESI). The role of the stator pucker in THI is discussed further in Section 3.3. These rapid THI events appear to occur too quickly relative to experimental observations that THI occurs on the microsecond timescale $[16,12]$. On one hand, these rapid THI events may be artifacts of the simulation methodology. On the other hand, the computational result could be reconciled with experiment if the kinetics of THI is bimodal, with a fast mode that happens on the picosecond timescale and a slow mode that happens on the microsecond or millisecond timescale. In the fast mode, photoisomerization and THI would happen in quick succession, and the motor would not have time to dissipate much of the kinetic energy from the excited to ground state hop before crossing the THI energy barrier. In the slow mode, however, the motor has time to fully equilibrate, with the kinetic energy from photoisomerization dissipated into many solute and solvent degrees of freedom before THI. If most trajectories dissipate their energy before completing THI, thus following the slow mode, then the fast mode would be a side process which does not contribute much to the overall kinetics observed in experiment. The experimental data available for these motors $[16,12]$ does not seem to allow one to determine whether THI in fact follows such bimodal kinetics.

As listed in Table 1, most replicates of all motors studied had not progressed to successful photoisomerization by the end of the simulations. Many of these replicates were still in the excited state, but there were also many that hopped to the ground state and fell back to the initial stable form, rather than progressing to the metastable form. These are unproductive replicates, in the sense that they would not lead to rotation of the motor. Quantum yields estimated from the fraction of productive replicates are on the same order of magnitude as reported experimentally for non- $\mathrm{MHz}$ alkene motors [13].

\subsubsection{Effects of thermostat and solvent viscosity}

The present simulations use the Langevin thermostat to regulate temperature (Section 2.1). With this method, a given temperature can be modeled by larger random forces ("kicks") and a larger drag coefficient, or by smaller kicks and a smaller drag coefficient. This scaling is controlled by the collision frequency parameter, which is connected with the viscosity. In the simulations described above, we used a collision frequency of $0.2 / \mathrm{ps}$, so that the kinetic energy released during photoisomerization dissipates into the bulk solvent on a several picosecond timescale, in agreement with experimental observations for a related photoexcited system [48]. For completeness, however, we also explored larger and smaller values of this parameter.

With a lower collision frequency of $0.02 / \mathrm{ps}$, corresponding to weaker kicks and lower viscous drag, the thermostat has less influence on the dynamics, so the kinetic energy added to the system by downhill motion on the excited state PES and by the subsequent hop to the ground state led to larger and longer-lasting rises in the effective temperature (kinetic energy) of the motors. Presumably 
because of the more extreme temperature rises, we observed more replicates that completed both photoisomerization and THI during the QM simulations. Interestingly, setting the collision frequency to zero, which decouples the motor from any heat bath and leads to a constant energy simulation, caused the photoisomerization success rate to drop, and a number of replicates ended in numerical failure, presumably due to motions too fast for the selected time-step. With a higher collision frequency of $2 / \mathrm{ps}$, the fraction of replicates that successfully hopped from the excited to the ground state within the simulation time was markedly reduced. Presumably, the increased viscosity slows rotation of the rotor toward the conical intersection, delaying return to the ground state.

\subsection{Optimization of force field parameters for overcrowded alkene motors}

The accuracy of classical MD depends on the accuracy of the force field used. To our knowledge, no specialized force field exists for alkene motors, except one reparametrized from OPLS for classical MD simulation of photoisomerization, rather than THI [21, 22]. In addition, our initial tests of GAFF2 force field yielded problematic results for these molecules, as detailed below. We therefore parameterized a specialized force field for each motor molecule by fitting to its unique QM potential energy surfaces and gradients, as detailed below.

\subsubsection{Motor $\mathrm{S}$ and Motor $\mathrm{N}$}

The QM energy surfaces of Motor S provided by TorsionDrive are smooth and well-converged, as evident in Fig 4A,B. The two deep minima in each energy surface correspond to the stable forms in Fig 1, and the shallower minima correspond to the metastable form. This clean separation of stable form and metastable form was not achieved by other plausible choices of reaction coordinate, such as when the central torsion angle was defined by atoms 16-15-14-13 or when using the stator pucker as a scan coordinate. The lack of perfect $180^{\circ}$ periodicity along the central torsion results from the geometric properties of the torsion and the nonplanarity of the rotor and stator. The motors rotate from right to left in these graphs (negative direction of the central torsion) because the metastable to stable transition along negative $\mathrm{x}$ direction has a moderate barrier height, whereas the metastable to stable transition along the positive $\mathrm{x}$ direction has an extremely large barrier.
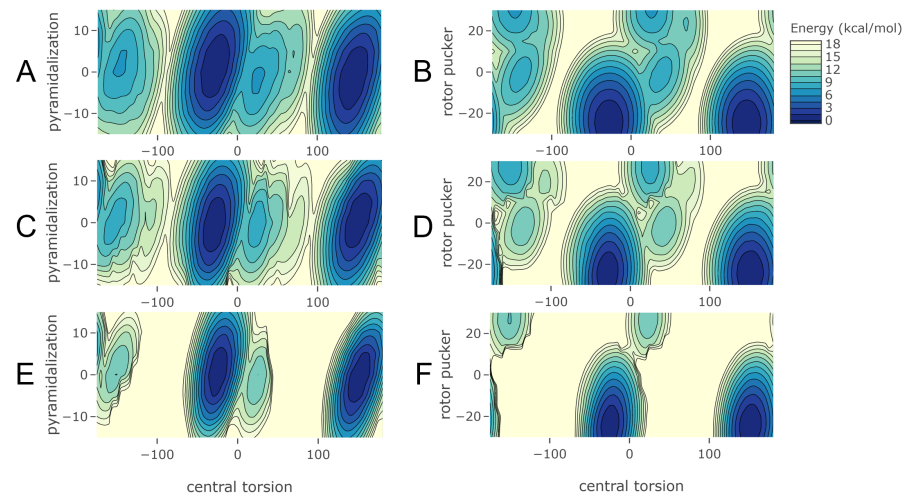

Figure 4: Potential energy surfaces of Motor S. A,B: quantum mechanical (QM) energy. C,D: energy from force field after fitting to QM. E,F: energy from GAFF2 force field before fitting to QM. The scanned coordinates are the central torsion angle, 18-15-14-10; the pyramidalization improper dihedral, 14-1-13-15; and the rotor pucker dihedral, 27-15-16-25, with atom numberings from Fig 1.The force field energy for each grid point was evaluated at the corresponding QM conformation without further relaxation.

The GAFF2 force field yields energy surfaces (Fig 4E,F) that deviate significantly from QM (Fig 4A,B). The energy barriers between minima are higher and wider than QM, and the metastable local minimum at around $(40,0)$ in QM (Fig 4B) is missed by GAFF2. Furthermore, we found that the unoptimized force field gave fundamentally incorrect conformations in MD simulations. For example, 
the stator heterocycle adopted a chair conformation, whereas experiments [16, 13] and QM suggest a boat conformation. GAFF1 yielded similar problems, and we expect other general force fields, if not tuned for alkene motors, to be inadequate in quantitative studies, if not qualitatively wrong.

Following optimization, the force field energy surfaces (Fig 4C,D) correctly represent all local minima and yield energy barriers similar to the QM results. It is notable that the optimization reduces the amplitudes of torsional energy terms associated with the central double bond and thus addresses the issue that the initial force field overestimated the barrier heights. In addition, optimization causes the various improper dihedral terms to have more varied amplitudes, ranging from essentially zero to $10.0 \mathrm{kcal} \mathrm{mol}^{-1}$, whereas all the GAFF2 amplitudes are $1.1 \mathrm{kcal} \mathrm{mol}^{-1}$. These changes presumably enable the optimized parameters to provide correct ring conformations. Note that only proper and improper dihedral terms were adjusted; other force field terms, such as bond-stretches and bond-angles, were left unchanged. For Motor N, the initial GAFF2 parameters again yield potential energy surfaces significantly different from the QM surfaces, and optimization of dihedral terms again yielded potential energy surfaces much closer to QM (Figure S1 in the ESI).

\subsubsection{Motor $\mathrm{O}$}

Optimization of force field parameters for Motor O posed additional challenges. Although optimization of force field dihedral parameters improves agreement with QM energy surfaces (Fig 5E,F), classical MD simulations with the resulting fitted parameters give no THI transitions at all for Motor O following optimization, implying an underestimation of the rate constant.
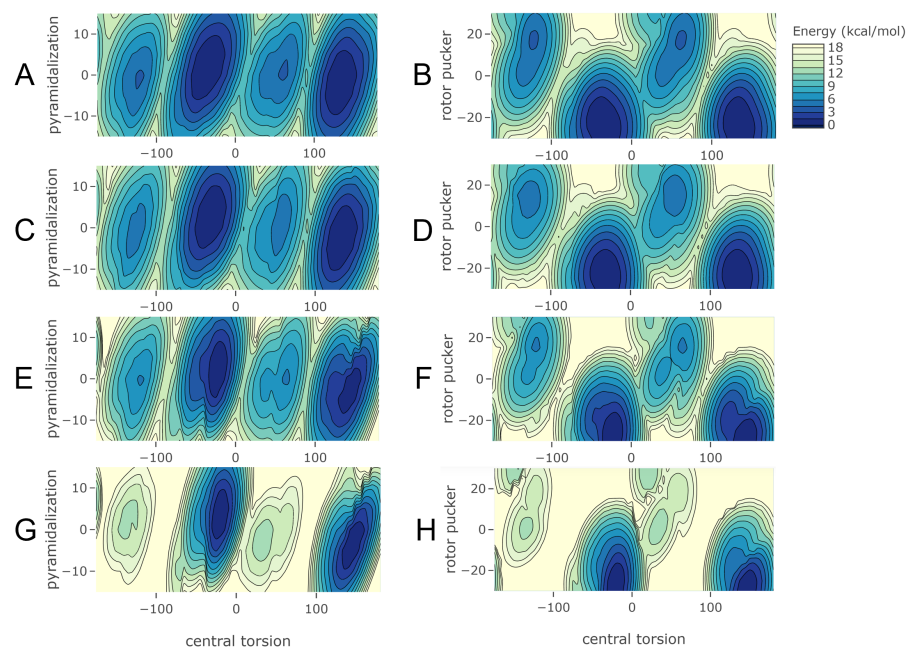

Figure 5: Potential energy surfaces of Motor O. A,B: quantum mechanical (QM) energy. E,F: energy from force field after fitting only dihedral terms to QM. C,D: energy from force field after tting dihedral terms, bond and angle terms, and terms of Buckingham potentials. G,H: energy from GAFF2 force field before fitting to QM. See Figure 4 for definitions of the scan coordinates.

We therefore tried optimizing not only dihedral terms for Motor $\mathrm{O}$, but also the $\sigma$ and $\epsilon$ values of each Lennard-Jones (LJ) term, as well as the force constants and equilibrium lengths of bond-stretch and angle-bend terms. These terms strongly affect the steric interactions between the stator and rotor during THI. To avoid nonphysically large changes in LJ terms, flat bottomed restraints were applied, to allowed maximal changes of $0.1 \AA$ in $\sigma$ and $0.015 \mathrm{~kJ} \mathrm{~mol}^{-1}$ in $\epsilon$. The resulting force field is softened, with $\sigma$ and $\epsilon$ smaller in most cases, and also reduced bond-stretch and angle-bend force constants. As expected, using the softened LJ terms in classical MD simulations allowed a greater THI rate that matches experiments. However, in this case the optimization algorithm made bond and angle force constants unphysically small, causing the molecule to be overly flexible during simulation. For example, the force constant of the central double bond, which is conjugated and thus should have a bond order between one and two, fell from the GAFF2 value of 855 to $312 \mathrm{~kJ} \mathrm{~mol}^{-1} \AA^{-2}$. Note that 
even the GAFF2 value is probably on the low side, because the force constant for a benzene $\mathrm{C}-\mathrm{C}$ bond, which also is of order between one and two, has been reported as $1124 \mathrm{~kJ} \mathrm{~mol}^{-1} \AA^{-2}$ [49].

We propose that the reason Motor $\mathrm{O}$ simulations face the dilemma of either underestimating the THI rate constant or using unrealistically soft bonds and angles is that the 12 th power repulsion of the LJ functional form is unrealistically steep [50] and therefore overestimates the van der Waals repulsion between the rotor and the stator during THI. For Motor O, among the scanned QM conformations, the closest distance between the rotor methyl $\mathrm{H}$ and the stator $\mathrm{H}$ on $\mathrm{C} 12$ is as close as $2.0 \AA$, well below the minimal-energy distance of $3.0 \AA$ for this interaction in the force field, and well into the range of distances where the 12 th power repulsion becomes problematic. Motors $\mathrm{S}$ and $\mathrm{N}$, in contrast, have a puckered and more flexible stator, and thus can alleviate this repulsion during THI, making the LJ repulsion acceptable for simulation of these motors.

We tested this explanation by replacing the LJ potential with the Buckingham potential [50], which has a less steep and hence more realistic exponential repulsion term. The initial Buckingham parameters were taken from Table III of Engler of coworkers [51] and the B cofficients were optimized without restraint. Bond-stretch and angle-bends were allowed to vary, subject to flat bottom restraints that prevented relative changes greater than 10\%. Optimization with the Buckingham potential led to improved agreement with QM, as shown in Fig 5C,D, without abnormally small force constants. Thus, the Buckingham potential may replace LJ potential for simulations of more congested molecules like Motor O. Unfortunately, support for the Buckingham potential in simulation software is currently limited. For example, it is not implemented in AMBER and, although it is implemented in GROMACS, it is not available in the GPU version. This made it impractical for use in simulations of Motor O at the timescale needed for the present study, so no further results are presented for this molecule. Future progress in software infrastructure is needed to enable Buckingham potential simulations at longer timescales.

\subsection{Classical MD simulation}

\subsubsection{Characterization of plain MD trajectories}

Thirteen classical MD simulations of Motor S were initiated from metastable form ground state conformations available at the end of the QM surface-hopping simulations (Section 3.1), and each was run for a total of $1 \mu \mathrm{s}$. Because the surface-hopping simulations of Motor $\mathrm{N}$ did not yield any metastable conformations we could use to initiate classical MD (Section 3.1), for this motor we instead initiated four $1 \mu \mathrm{s}$ classical MD simulations, one from each of the four metastable substates identified by the MSM (Section 3.3.2). No obvious pathologies were observed in the course of these simulations for either motor, and several interesting trends were observed.

First, 10 of the 13 simulations of Motor S and one of the four simulations of Motor N completed THI during their $1 \mu \mathrm{s}$ duration. The full rotation process, consisting of excited state dynamics, decay to the ground state, ground state dynamics within the metastable form, and THI, is captured by the concatenation of a QM surface-hopping trajectory and its subsequent classical MD trajectory for Motor S in video mndo-amber.mp4 in the ESI.

Intriguingly, just as seen for THI transitions during the QM surface-hopping MD simulations (Section 3.1), every THI transition in the classical simulations was immediately preceded by flipping of the stator pucker to that of the target stable form. This is not visible in video mndo-amber.mp4 because of the long time interval between frames, which was chosen to allow viewing of the full rotation process. Therefore, in videos amber-SnearTHI.mp4 and amber-NnearTHI.mp4 of the ESI, we zoom in to animate the details near the THI transition. Figure 6 shows the time series of the key coordinates, i.e. the central torsion angle and puckers, to illustrate the transitions in amber-SnearTHI.mp4. The first event is a sharp change in the pucker of the stator to that of the final stable form, along with a transient backward rotation of the rotor relative to the stator. This is followed by a sharp forward rotation of the rotor to reach the stable form. Meanwhile, the rotor pucker fluctuates rapidly between axial and equatorial until the stator pucker changes, at which point the rotor pucker becomes locked in the equatorial conformation until the rotor rotates to the stable form, at which point the rotor pucker becomes locked in the axial form. Sections 3.3.2 and 3.3.4 provide further details regarding the THI mechanism and kinetics. 


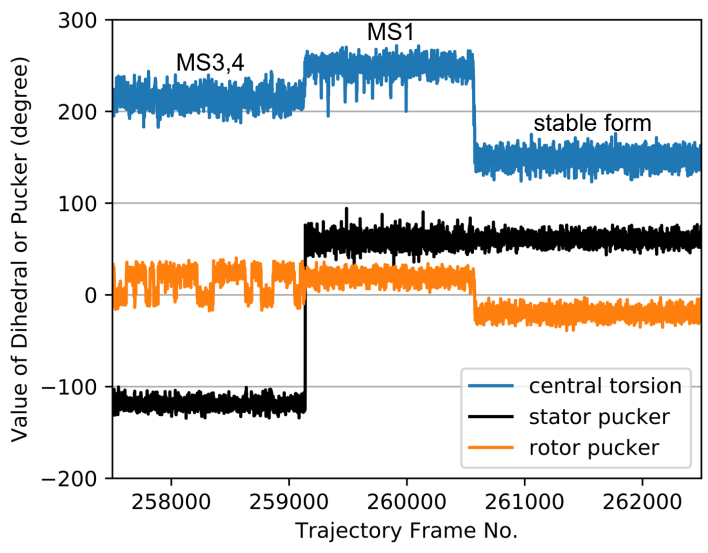

Figure 6: Time series of the central torsion angle and puckers before, during, and after a THI transition simulated by classical MD. Forward rotation of the rotor relative to the stator corresponds to a decrease in the central torsion angle. The puckers are defined so that positive/negative rotor pucker mean equatorial/axial, respectively, and positive/negative stator pucker mean the same/opposite pucker as final stable form, respectively. Labels indicate which conformational state (Section 3.3.2) is occupied at each step.

\subsubsection{Substate analysis of the metastable form via Markov state modeling}

The conformational substates of the metastable form of Motor S and their transition network were obtained in the form of a Markov state model (MSM), through analysis of the thirteen classical MD trajectories. Representative conformations of the four resulting substates are depicted in Fig 7, along with circles indicating their steady state probabilities estimated from the MSM transition matrix. The ESI furthermore provides Cartesian coordinates of ten sample conformations for each Markov state. The transition time constants, i.e. inverse rate constants, are shown with black arrows representing the associated transitions between substates. Orange arrows represent photoisomerization. As noted in Sections 3.1 and 3.3, the transition to the stable form is always preceded by a flip of the stator pucker to that of the target stable form. The MSM groups the conformations with this pucker into a single substate, called metastable substate 1 (MS1), and an additional red arrow goes from MS1 to the stable form in Fig 7. The 10ns time constant for this transition is estimated from the simulation time spent in MS1 for all 13 trajectories, divided by the number of THI transitions observed. The large arrow from the metastable form as a whole to the stable form is labeled with rate constant computed by WESTPA simulation (Section 3.3.4). The states and labelled arrows in Fig 7 define a complete kinetic model of the whole motor rotation process, which is analyzed quantitatively in Section 3.3.3.

Our kinetic model in Fig 7 indicates that MS1, despite its low population, is a necessary stop on the path from the metastable to the stable form. Thus, THI can be viewed as taking place in two consecutive steps: change of the stator pucker to that of the final stable form, followed by rotation of rotor, relative to the stator, to complete THI. Metastable substates 3 and 4 (MS3, MS4), which hold the majority of the population, are similar in conformation, as they only differ by their rotor pucker, and they interconvert quickly. Metastable substate 2 (MS2) is the closest to the stable form in terms of its central torsion angle (defined as e.g. 18-15-14-10 or 17-18-2-1), but the model implies that MS2 is actually the furthest kinetically from the stable form. This finding highlights the potential drawback of choosing a single geometric feature as the reaction coordinate: using only the central torsion angle as the reaction coordinate risks giving the incorrect impression that a motor in MS1, MS3, or MS4, needs to go to MS2 as the obligatory last step before reaching the stable form. Exploring mechanisms by unconstrained MD simulations, instead of exploring along predefined reaction coordinates, helps avoid such problems. These high dimensional MD trajectories are free of bias or information loss from inexact assumptions based on low dimensional intuitions, yet the trajectories can be projected to informative lower dimensional pictures based on analysis of structure and kinetics.

We also generated a MSM for Motor N from four $1 \mu$ s classical MD trajectories, each started from a 


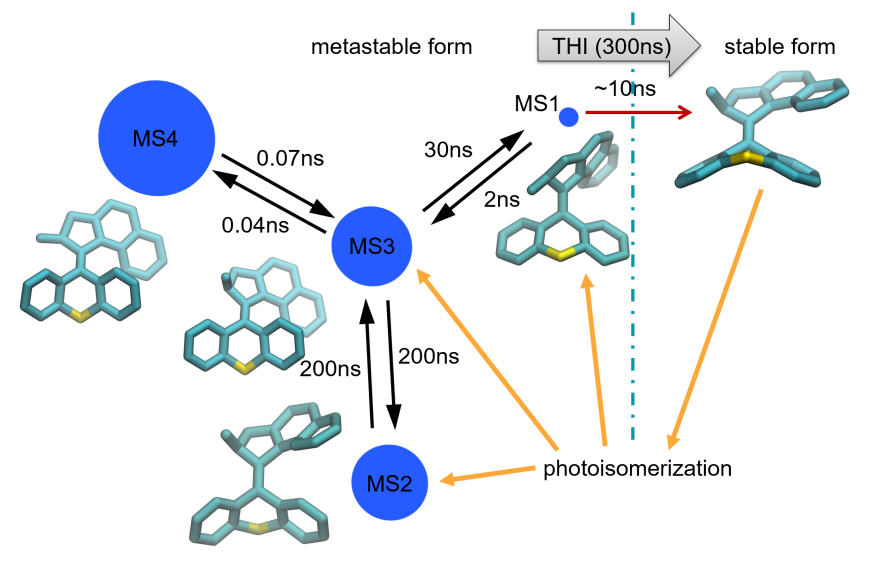

Figure 7: Kinetic model of Motor S rotation at 300K. Each metastable substate is represented as a circle of area proportional to steady state probability, along with a representative 3D structure. Black arrows are drawn for each nonzero transition matrix element of the MSM. Numbers on the arrows are corresponding transition time constants calculated with the MSM. A red arrow represents THI transitions that appear to always go through MS1 in classical MD; the associated time constant is calculated as the mean wait time in MS1 before transition to stable form. The gray arrow indicates the overall THI process and gives the overall THI rate constant estimated with WESTPA (Sec 3.3.4). Additional orange arrows are drawn to represent photoisomerization to metastable substates seen at the end of the QM surface-hopping MD runs.

conformation similar to one of the metastable substates of Motor S. The MSM was again constructed from the time series of all the dihedrals with associated force field terms, except those involving at least one atom of the -COOtBu group. The resulting model (Fig 8) is very similar to that of Motor S.

Prior studies using static QM methods also delineated metastable substates of a motor very similar to the present Motor S [14, 15]. Although the conformations of those substates are similar to the ones reported here, the prior studies proposed a different pathway to the stable form. Rather than going through the relatively high energy MS1 as found here, their pathway goes to the stable form through the lower energy MS2. Potential explanations for the difference between the prior results and those presented here include the fact that the motor molecule studied previously had an extra methoxy group, relative to Motor S; the prior studies' use of high-level QM calculations throughout; the challenge of finding all relevant transition states by QM geometric optimization in the prior approach; and our use of a dynamical, rather than a static, method.

\subsubsection{Steady state kinetics of Motor S}

The diagram in Figure 7 offers a kinetic model of Motor $\mathrm{S}$ and includes estimated rate constants for many of the interstate conversions. Although we do not have estimated rate constants for photoisomerization of the stable form into the various metastable substates (orange arrows), the properties of the motor may be explored by assuming a range of values for these missing quantities and solving for the steady state probabilities and rates, as detailed in the Appendix A. (The Appendix also considers the consequences of backward photoisomerization from the metastable to the stable form, which is omitted here.) The present results correspond to experiments where the motor is continuously illuminated while at a temperature that allows THI $[18,52]$, so that the motor may rotate continuously.

We first assume that the photoisomerization process deposits the system into each of the four metastable substates in the same proportions as observed in the QM surface-hopping MD calculations (Section 3.1), i.e., MS1:MS2:MS3:MS4=7:2:4:0. Thus, with an overall photoisomerization rate constant of $k_{\text {photo }}$, the rate constant for arriving in MS1 is $\frac{7}{13} k_{\text {photo }}$, and so on. The steady state probabilities of the system's states are plotted as a function of $k_{\text {photo }}$ in Fig $9 \mathrm{~A}$. At low $k_{\text {photo }}$, the stable form has a probability near unity and the metastable substates have probabilities near zero. As $k_{\text {photo }}$ rises above $\sim 10^{5} \mathrm{~s}^{-1}$, the probability begins to shift from the stable form to the various metastable substates; and 


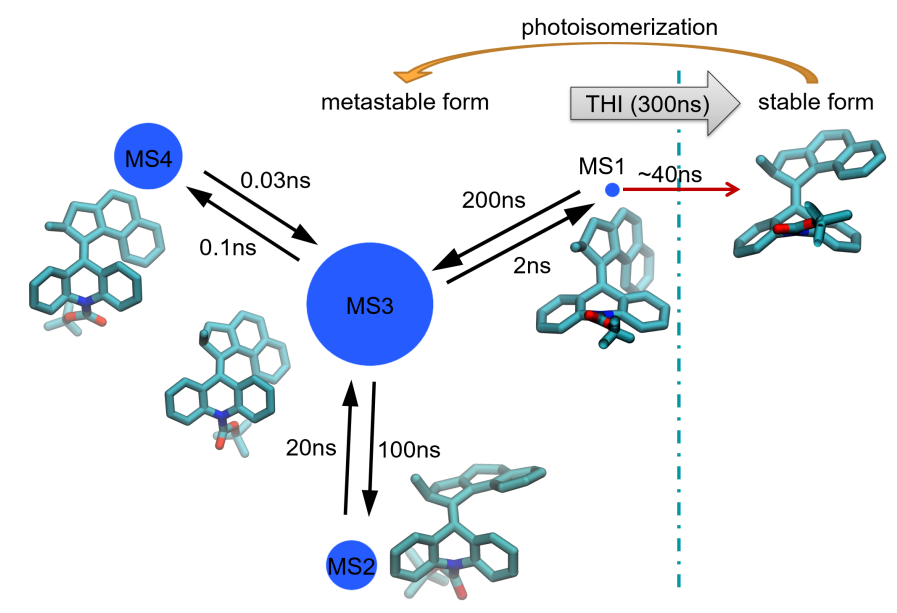

Figure 8: Kinetic model of motor $\mathrm{N}$ rotation at 300K. Each metastable substate is represented as a circle of area proportional to steady state probability, along with a representative 3D structure. Black arrows are drawn for each nonzero transition matrix element of the MSM. Numbers on the arrows are corresponding transition time constants calculated with the MSM. A red arrow represents THI transitions that appear to always go through MS1 in classical MD; the associated time constant is calculated as the mean wait time in MS1 before transition to stable form. The gray arrow indicates the overall THI process and gives the overall THI rate constant estimated with WESTPA (Sec 3.3.4), and the orange arrow indicates photoisomerization

a plateau is approached at $k_{\text {photo }} \approx 10^{8} s^{-1}$, where the stable form probability nears zero.

The motor's rotation rate (Figure 9B, orange curve) has a similarly sigmoid dependence on $k_{\text {photo }}$. The asymptotic maximal rotation rate, $1.5 \mathrm{MHz}$, is approached as the stable form spends a negligible amount of time waiting for photoisomerization, so that THI is rate-limiting. The $k_{\text {photo }}$ at which the rate is $50 \%$ of its asymptotic maximum, is $k_{\text {photo,50 }}=1 \times 10^{6} \mathrm{~s}^{-1}$.

Interestingly, the maximal rotation rate (Figure 9B) does not depend dramatically on the ratio of metastable substates immediately following photoisomerization. Assuming that photoisomerization generates only MS1 (MS1:MS2:MS3:MS4=1:0:0:0) increases maximal rotation rate by about 10\% (Fig 9B, blue), because MS1 is close to completing THI (Fig 7). Conversely, assuming that photoisomerization generates only MS2 (MS1:MS2:MS3:MS4=0:1:0:0) reduces maximal rotation rate by about $15 \%$, because MS2 must go through a relatively slow conversion to MS3 before going to MS1 and then to stable form (Fig 7). The insensitivity of the rotation rate to the details of photoisomerization supports the approach taken in this study, which focuses on THI process with less comprehensive modeling of photoisomerization. However, the rotation rates of other light-driven motors may not be so insensitive to the distribution of metastable substates generated by photoisomerization. For example, if a motor had the same transition network and time constants as in Fig 7 except for a much longer time constant to go from MS1 to MS3, its rotation rate would be more sensitive to the distribution of metastable substates generated by photoisomerization, as shown in Figure 16 of Appendix A.

It is worth clarifying that, although the photoisomerization process starting from the FranckCondon point is very fast (on the picosecond timescale), the photoisomerization rate constant is determined by a longer timescale, i.e., the wait time for photoexcitation. This wait time is determined not just by the intrinsic properties of the motor, but also by experimental settings, such as the intensity and spectrum of the light. Indeed, approaching the maximum rotation rate in this figure may require light intensities too high to be readily generated in experiments, as previously emphasized [52]. The need for such high intensities traces to the low molar absorptivities of these compounds, about 10,000 $\operatorname{molar}^{-1} \mathrm{~cm}^{-1}$ ) [16]. 

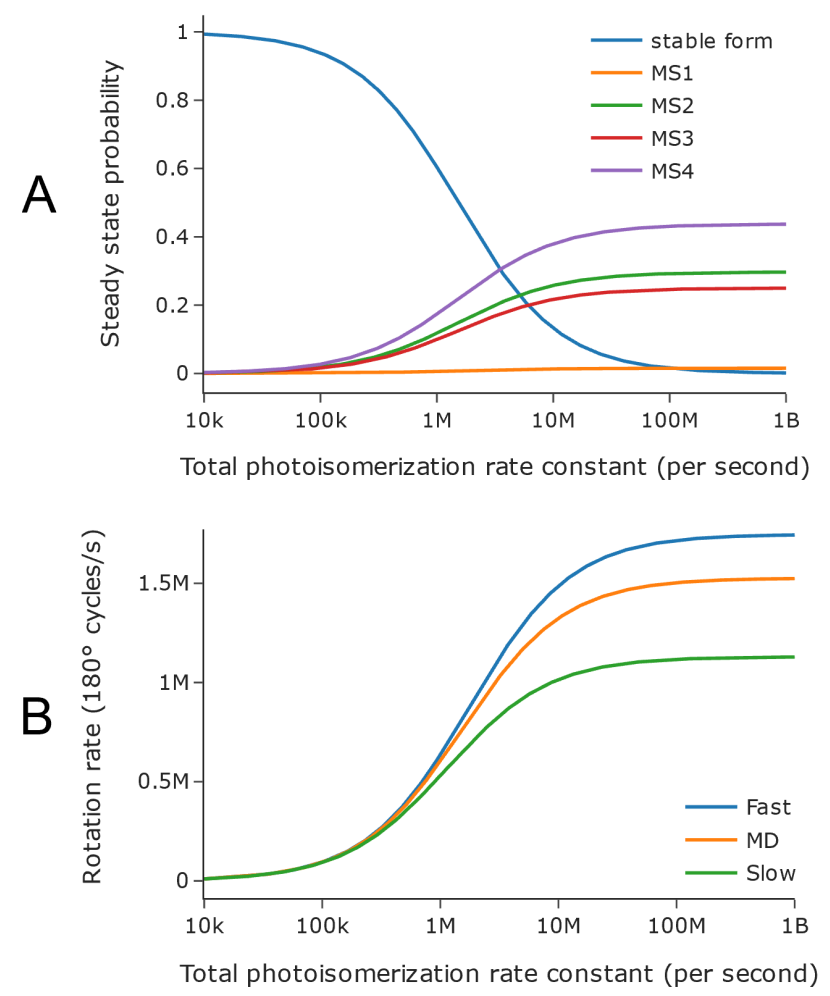

Figure 9: Steady state kinetics of Motor S at 300K. A: dependence of the steady state probabilities on the total photoisomerization rate. B: dependence of steady state rotation rate of the motor on the total photoisomerization rate, where the three curves correspond to three different distributions of metastable substates generated by photoisomerization: the distribution that leads to the fastest rotation (MS1:MS2:MS3:MS4=1:0:0:0), the distribution that leads to the slowest rotation (MS1:MS2:MS3:MS4=0:1:0:0), and the distribution inferred from our surface-hopping MD trajectories (MS1:MS2:MS3:MS4=7:2:4:0). In A, the distribution from MD is used.

\subsubsection{THI rate constant from weighted ensemble MD simulations}

THI is often the rate-limiting rotation step of the alkene motors, so its rate constant is a critical performance metric. Although some of the classical MD simulations discussed above led to THI transitions, more are needed for a precise estimate of the THI rate constant. We therefore used weighted ensemble simulations in the software package WESTPA to sample THI transitions.

For WESTPA to facilitate the key conformational transitions, progress coordinates must be defined that clearly distinguish the slowly interconverting states. We chose two-dimensional progress coordinates - a rotor-stator rotation angle (17-18-2-1) and the stator pucker - that clearly distinguish among the slowly interconverting metastable substates MS1, MS2, and MS3/MS4, as well as the stable form, as shown in Fig 10. WESTPA calculations were run for both Motor S and Motor N, using the same starting points as their respective plain classical MD simulations (Section 3.3.1). As shown in Fig 11 and Fig 12, the final computed THI rate constants are within an order of magnitude of the corresponding experimental results, as well as the modeling results in Section 3.3.3.

In experimental studies, increasing the solvent viscosity significantly decreased the THI rate constant [53]. Our simulations reproduced this trend (Fig 13) by comparing THI rate constants from simulations using different Langevin collision frequencies, as the largest collision frequency, 10/ps, which simulates the largest effective viscosity, resulted in a smaller THI rate constant than $1 / \mathrm{ps}$ and $0.1 / \mathrm{ps}$. The smallest collision frequency, $0.01 / \mathrm{ps}$, also appears to decrease the THI rate, in agreement with a prior study about how collision frequency influences conformational sampling of a peptide [54]. Overall, however, the computed THI rate constant is not very sensitive to the somewhat arbitrary 


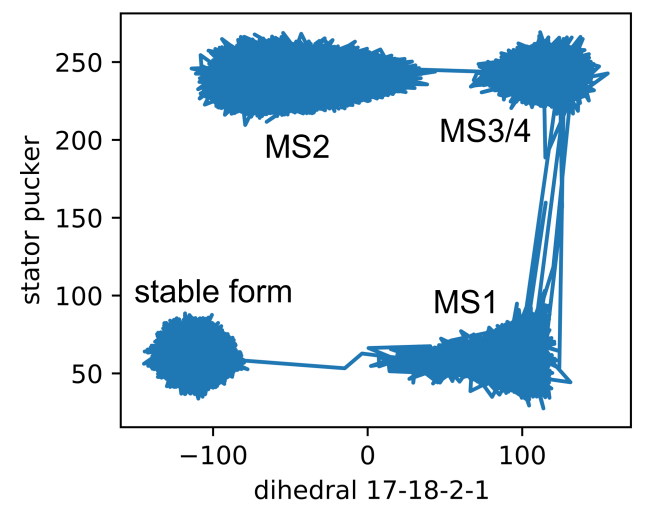

Figure 10: Projection of one of the 13 plain MD simulations onto the two progress coordinates used in weighted ensemble simulation of Motor S. Clusters are labelled according to their conformational states (Fig 7).

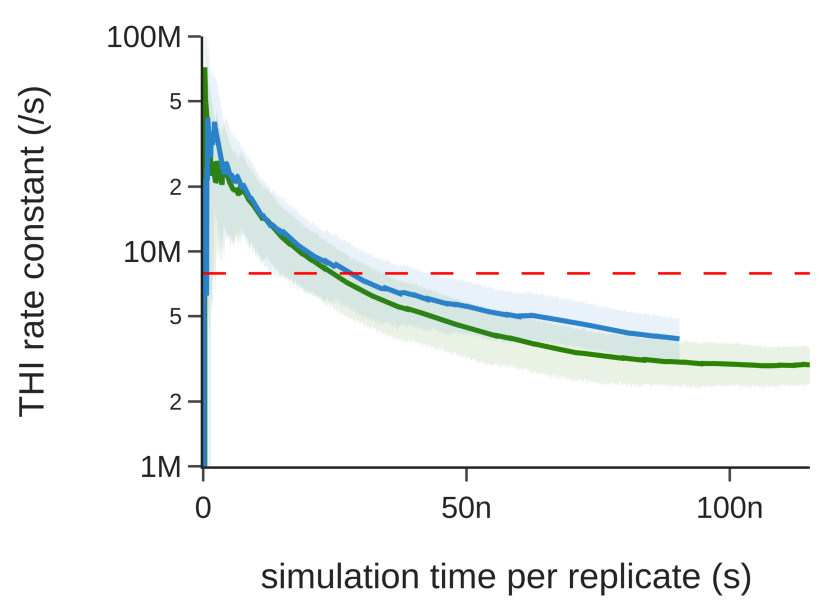

Figure 11: THI rate constant of Motor S computed with WESTPA, as a function of simulation time per replicate, with shading to indicate $95 \%$ bootstrapping confidence interval. Results are shown for two runs with identical settings except different random number seeds. Dashed red line indicates experimental rate constant at $300 \mathrm{~K}, 7.9 \times 10^{6} \mathrm{~s}^{-1}$, read from Arrhenius plots of Klok et al.[16].

choice of collision frequency.

\subsubsection{Free energy available from THI}

The results presented above focus on the mechanisms and kinetics of the motor rotation. Here, we present results for the free energy difference between the metastable form and the stable form, and discuss the implications of this quantity as a performance metric. We used umbrella sampling to obtain the PMF for rotation around the central double bond, defined here by torsion 43-15-14-29. As shown in Fig 14 for Motor S, the metastable form is separated from the stable form by a free energy barrier. Integration along the PMF (Section 2.2) gives a favorable free energy release of $7.8 \mathrm{kcal} \mathrm{mol}^{-1}$ on going from the metastable to the stable form (right to left in the figure). Note that the height and width of the barrier makes this free energy difference insensitive to the precise choice of boundary between the stable form's free energy well on the left and the metastable form's free energy well on the right. The corresponding PMF for Motor $\mathrm{N}$ is similar in shape (Fig 15), and the free energy difference is slightly greater, at $8.8 \mathrm{kcal} \mathrm{mol}^{-1}$. Thus, the available free energy from THI is similar to the standard free energy released by hydrolysis of ATP, the cell's energy currency, $\sim 8 \mathrm{kcal} \mathrm{mol}^{-1}[55]$.

The torque available from THI, averaged over the $180^{\circ}$ rotation cycle of the motor, can be estimated 


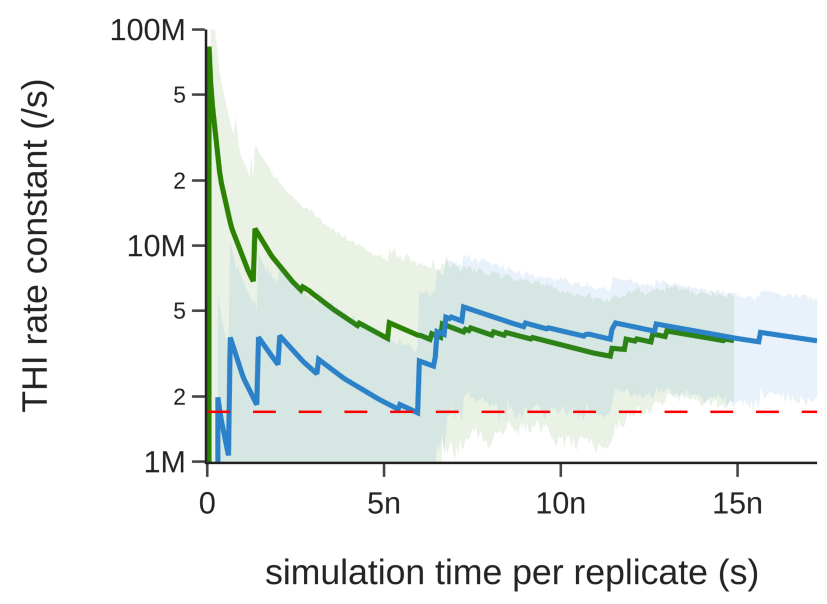

Figure 12: THI rate constant of Motor N computed with WESTPA, as a function of simulation time per replicate, with shading to indicate $95 \%$ bootstrapping confidence intervals. Results are shown for two runs with identical settings except different random number seeds. Dashed red line indicates experimental rate constant at $300 \mathrm{~K}, 1.7 \times 10^{6} \mathrm{~s}^{-1}$, from [12].

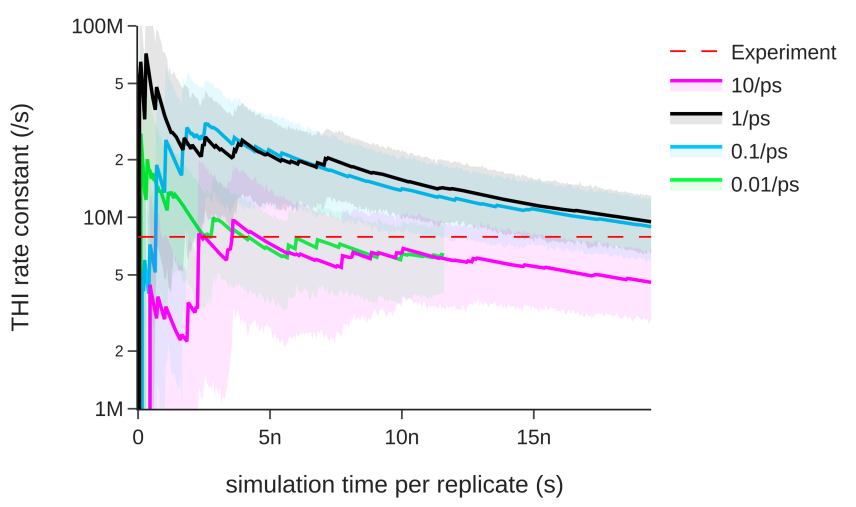

Figure 13: THI rate constants of Motor S computed with WESTPA, as a function of simulation time per replicate, for four different collision frequences. Shaded areas indicate $95 \%$ bootstrapping confidence intervals. Dashed red line indicates experimental rate constant [16].

as the free energy change, $7.8 \mathrm{kcal} \mathrm{mol}^{-1}$, divided by $\pi$ radians, which gives a torque of about $18 \mathrm{pN} \mathrm{nm}$. This may be compared with $40 \mathrm{pN} \mathrm{nm}$ for the highly evolved F1-ATPase motor [56]. Note that this analysis omits any contribution from the photoisomerization step, which also runs in the forward direction and could in principle contribute more to the average torque.

We tested the numerical reliability of the free energy evaluations by rerunning all the simulations with different random number seeds, and obtained free energy differences within $0.3 \mathrm{kcal} \mathrm{mol}^{-1}$ of the original results. In addition, rerunning with umbrella sampling spring constant of 100 instead of 200 $\mathrm{kj} \mathrm{mol}^{-1}$ affected the result by less than $0.3 \mathrm{kcal} \mathrm{mol}^{-1}$, whereas varying WHAM parameters and the integral bounds within reason gave even smaller changes. Thus, the numerical uncertainties in these results appear to be well below $1 \mathrm{kcal} \mathrm{mol}^{-1}$, and we expect that the force field is a larger source of error than the numerical uncertainty. Furthermore, these force field-based free energy differences are close to those previously obtained by purely QM methods for a similar motor [15].

The maximal power available to drive an external load is less than the product of the maximal rotational rate and the free energy change, because loading the motor decreases its rotation rate. To get a sense for the power available from Motor S, we used WESTPA to recalculate the THI rate constant in the presence of a constant external torque along torsion angle 43-15-14-29. The external torque opposes 


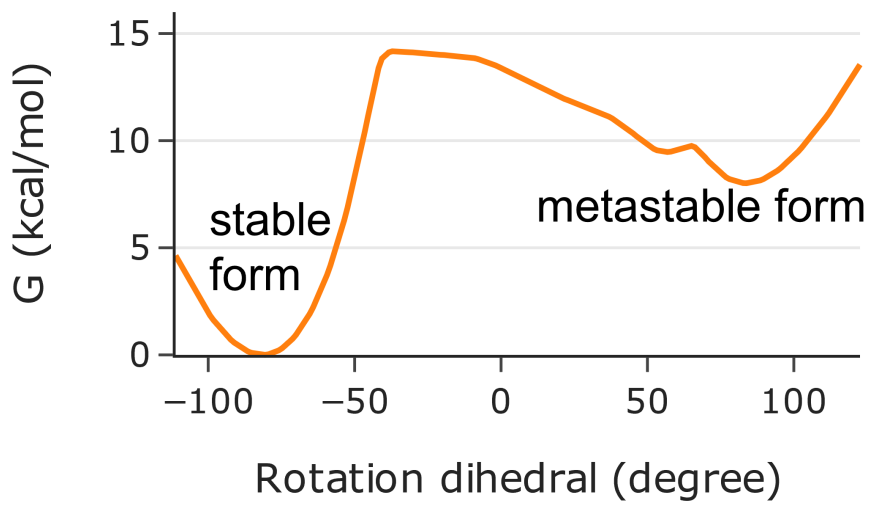

Figure 14: Computed Gibbs free energy as a function of rotation dihedral (43-15-14-29) for Motor S.

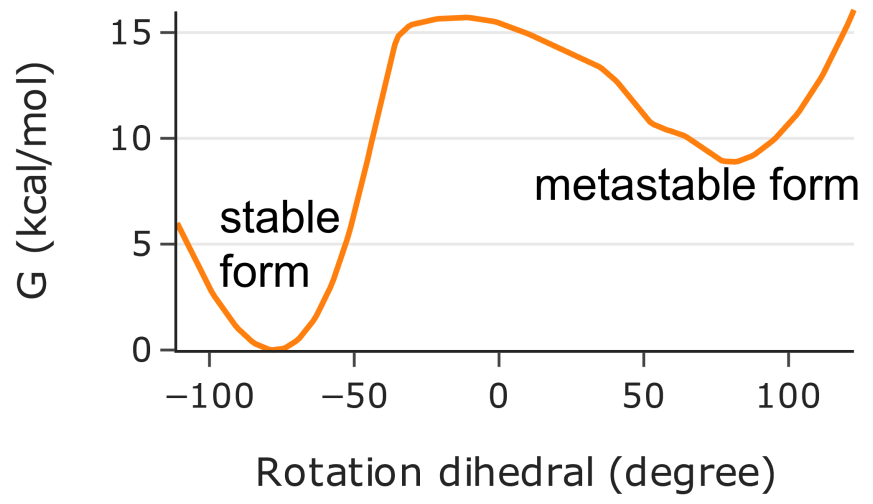

Figure 15: Computed Gibbs free energy as a function of rotation dihedral (43-15-14-29) for motor N.

forward rotation, with an energy difference (load) of $3.4 \mathrm{kcal} \mathrm{mol}^{-1}$ between the metastable form and the stable form. Adding this load dropped the THI rate constant to $1 \times 10^{5} \mathrm{~s}^{-1}$ (Figure S2 in the ESI), about an order of magnitude below the unloaded motor (Fig 11). This loaded THI rate constant yields a power output of about $4 \times 10^{5} \mathrm{kcal} \mathrm{mol}^{-1} \mathrm{~s}^{-1}$, when not bottlenecked by photoisomerization [52]. This potential power output is higher than typical biochemical reactions, because even fast enzymes, such as ATP synthase $\left(1 \times 10^{3} \mathrm{~s}^{-1}[57]\right)$ and catalase $\left(1 \times 10^{4} \mathrm{~s}^{-1}[58]\right)$, cycle much more slowly.

It is worth emphasizing that the $\sim 8 \mathrm{kcal} \mathrm{mol}^{-1}$ of free energy available from THI is only a fraction of the $80 \mathrm{kcal} \mathrm{mol}^{-1}$ energy provided by a photon from the $355 \mathrm{~nm}$ laser used in the experiments [16]. As a consequence, it is of interest to consider how to improve the energy efficiency. One possibility may be to modify the structure in a manner that makes the metastable form less stable, without degrading other key parameters. This would probably bring the additional benefit of lowering the THI barrier and thus increasing the rotation rate. It might also be possible to collect power not only from THI but also from the photoisomerization step, as noted above.

A previous study [53] obtained a far lower estimate of the work available from THI of Motor S. Using structures from QM calculations, the authors found that the stable form has a solvent-excluded volume $7 \AA^{3}$ larger than that of the metastable form, and they computed the pressure-volume (PV) expansion work associated with this expansion, which comes to only $0.006 \mathrm{kcal} \mathrm{mol}^{-1}$. We obtained a similar volume change, $5 \AA^{3}$, for this step, by examining the time-series of system volumes in our explicit solvent simulations run at room pressure. However, it is the rotary work available from these motors, not the PV work that should be of interest. This may be understood by considering that capturing work done by the motor involves coupling an external load to the angle between the stator and rotor, not to a change in volume. More broadly, it is also worth noting that the Gibbs free energy change is the maximal non-PV work in a constant-temperature, constant-pressure, closed system. 


\section{Conclusions}

We have studied a class of fast, light-driven overcrowded alkene-based molecular motors with a novel multiscale computational framework that combines quantum mechanical (QM) surface-hopping molecular dynamics (MD) simulations with classical MD simulations. The main conclusions are as follows.

1. Trajectories of the rotation process (videos in the ESI) give a rich picture of the full rotation dynamics of these motors.

2. Kinetic models derived from simulation trajectories provides a detailed rotation mechanism (Fig 7); notably,

- A flip of the stator pucker, generating metastable substate 1 (MS1), immediately precedes and appears to enable THI transition to the stable form.

- The maximal rotation speed can be calculated from the model and depends on the substate interconversion rates, as well as the distribution of metastable substates generated by the photoisomerization process.

3. Experimentally measured THI rate constants are replicated to within an order of magnitude using weighted ensemble MD simulations with optimized force field parameters. Thus, this technology may be useful in designing new molecular motors with desired rotation rates.

4. The maximal available work from THI is estimated as $\sim 8 \mathrm{~kJ} \mathrm{~mol}^{-1}$, which corresponds to an average torque of $\sim 18 \mathrm{pN} \mathrm{nm}$. Simulated under half load, the motor still has a high THI rate constant $1 \times 10^{5} \mathrm{~s}^{-1}$ and the maximal power output is about $4 \times 10^{5} \mathrm{kcal} \mathrm{mol}^{-1} \mathrm{~s}^{-1}$. These magnitudes are similar to and sometimes higher than biological motors, such as the F1-ATPase.

5. Classical MD simulations with a widely used general force field gave incorrect conformations and dynamics. Far better results were obtained after fitting selected force field parameters to QM potential energy surfaces.

6. For Motor O, with its planar stator, the Lennard-Jones term appeared inadequate to model van der Waals interactions during THI, probably due to its unrealistically steep repulsion potential. The less steep and hence more realistic Buckingham potential provided better results.

The computational and theoretical framework used here is modular, so future studies could use refined forms of each component method. For example, photoisomerization could be modeled with higher level QM techniques, such as Ab Initio Multiple Spawning methods [59], and the classical simulations could use improved functional forms of the force field, such as a more realistic van der Waals potential or a more detailed description of torsional energy profiles. The present framework can also be extended to other classes of fast light-driven molecular motors, especially those that share the same overcrowded alkene core $[60,25]$

\section{Conflicts of interest}

MKG has an equity interest in and is a cofounder and scientic advisor of VeraChem LLC.

\section{Acknowledgements}

We thank Drs David Slochower, Jeffry Setiadi, Lee-Ping Wang, and Adrian Roitberg for helpful discussions, and Drs Jie Liu, Axel Koslowski and the late Walter Thiel for their help with MNDO.

MKG acknowledges funding from National Institute of General Medical Sciences (GM061300). These ndings are solely of the authors and do not necessarily represent the views of the NIH. 


\section{Appendix A: derivation of steady state rotation rate under con- tinuous excitation}

When the motor molecules are simultaneously excited by light and heated, they will rotate cycle after cycle. A steady state can be reached under constant conditions, and here we derive the steady state probabilities of all the conformational states drawn in Fig 7, and the corresponding probability flux, i.e., the steady state rotation rate. These results were used to produce Fig 9.

The MSM transition matrix associated with Fig 7 can be viewed as a discretization of continuoustime differential equations of chemical kinetics, written below [61].

$$
\begin{gathered}
\frac{d p_{s}}{d t}=k_{1 s} p_{1}-\left(k_{s 1}+k_{s 2}+k_{s 3}\right) p_{s} \\
\frac{d p_{1}}{d t}=k_{s 1} p_{s}+k_{31} p_{3}-k_{1 s} p_{1}-k_{13} p_{1} \\
\frac{d p_{2}}{d t}=k_{s 2} p_{s}+k_{32} p_{3}-k_{23} p_{2} \\
\frac{d p_{3}}{d t}=k_{s 3} p_{s}+k_{23} p_{2}+k_{13} p_{1}-k_{32} p_{3}-k_{31} p_{3} \\
p_{4}=p_{3} \frac{k_{34}}{k_{43}}
\end{gathered}
$$

The last equation posits rapid equilibrium between MS3 and MS4, based on the much faster interconversion time between them. Here, $p$ indicates a probability, an s subscript indicates the stable form and an integer subscript indicates the corresponding metastable substate, and the rate constant from state a to $\mathrm{b}$ is denoted $k_{a b}$. Setting the derivatives to zero and solving these equations with the constraint $p_{1}+p_{2}+p_{3}+p_{4}+p_{s}=1$ gives steady state probabilities $\pi_{1}, \pi_{2}, \pi_{3}, \pi_{4}, \pi_{s}$ as functions of the rate constants. Then the steady state rotation rate (flux) equals $k_{1 s} \pi_{1}$. With good MSM discretization, each rate constant in the MSM should match the corresponding rate constant in these differential equations, so we borrow the rate constants from Fig 7 to use here. Scanning over a range of photoisomerization rate constants and considering different distributions of metastable substates generated by photoisomerization produce Fig 9, as detailed in Section 3.3.3.

We anticipated that the motor rotation rate would become more sensitive to the distribution of metastable substates generated by photoisomerization if $k_{13}$ was smaller. To test this, we reduced $k_{13}$ by a factor of 10000 , while leaving other rate constants unchanged. As shown in Fig 16, the rotation rate now is, in fact, highly sensitive to the distribution of metastable substates generated by photoisomerization. Intuitively, the drop in $k_{13}$ means that virtually all trajectories initiated in MS1 will quickly undergo THI, rather than being delayed by going to MS3. A Jupyter notebook in our Github repository contains the source code for this calculation and plotting.

In the above analysis, we have omitted backward photoisomerization, i.e., photoisomerization from $\mathrm{B}$ to $\mathrm{A}$ or from D to $\mathrm{C}$ (Fig 1), because including this would have required making additional arbitrary assumptions about the rate constants for backward photoisomerization from each of the metastable substates. This omission should not be problematic when the illumination is weak enough and/or the temperature is high enough, so that the backward photoisomerization rate is small compared to the forward THI rate. However, in settings where backward photoisomerization is substantial relative to forward THI, we expect the model given by the above equations to underestimate the steady state probabilities of the stable form, and overestimate the rotation rate. This is because backward photoisomerization provides a nonproductive channel by which probability can drain away from the metastable form instead of going through forward THI. Although the quantitative details will depend on the backward photoisomerization rate constants from each of the metastable substates, the maximal rotation rate approached asymptotically with increasing levels of illumination should be approximately

the maximal rotation rate from the present model multiplied by the probability of the metastable form in the photostationary state, which is usually on the order of $20 \%$ for the similar Motor O [53]. A 


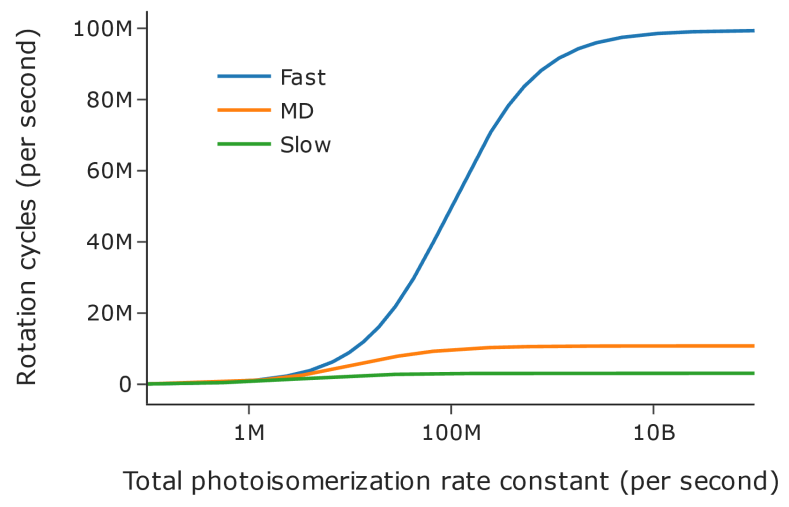

Figure 16: Dependence of the motor rotation rate in steady state on the total photoisomerization rate, assuming 10000 times smaller $k_{13}$ than its value obtained from MSM and used in Figure 9. The three curves correspond to three different distributions of metastable substates generated by photoisomerization: the distribution that leads to the fastest rotation (MS1:MS2:MS3:MS4=1:0:0:0), the distribution that leads to the slowest rotation (MS1:MS2:MS3:MS4=0:1:0:0), and the distribution inferred from our surface-hopping MD trajectories (MS1:MS2:MS3:MS4=7:2:4:0).

prior study [52] provides valuable detail on how backward photoisomerization affects the steady state rotation rate, but without resolving the metastable form into substates.

\section{Appendix B: selection of reaction coordinates}

The rotation of the rotor relative to the stator is the defining feature of these motors. The most common way to describe this rotation is a torsion angle defined with 4 consecutive atoms around the central double bond, such as 16-15-14-13. However, we found that such dihedrals are insensitive to the ground state conformational changes, as they remain rather close to either $0^{\circ}$ or $180^{\circ}$. Instead, non-sequential dihedrals involving two rotor and two stator atoms, such as 18-15-14-10 and especially 43-15-14-29 or 17-18-2-1, show larger and more definitive changes as the rotor naphthalene atoms snap past the stator benzene atoms to complete THI. The Cremer-Pople definition of the stator pucker [62] provides a valuable second reaction coordinate.

Upon excitation, the central double bond becomes more single bond-like, leading to carbon pyramidalization; in contrast, during ground state simulations we found the degree of pyramidalization to be small. Consequently, pyramidalization coordinate is only useful for characterizing photoisomerization or the whole rotation cycle including photoisomerization. Although the rotor pucker was a useful reaction coordinate for the QM potential energy surfaces (Fig 4), it was a less informative coordinate than the stator pucker when focusing on THI, except for Motor O, whose stator is planar. One reason is evident in Fig 7, which shows that interconversion between different rotor puckers (MS3 and MS4) is much faster than interconversion between different stator puckers.

It is worth noting that a reaction coordinate based on an atom that is more rigidly linked to the rest of the molecule, such as an sp2 carbon, is generally better than one based on a more mobile atom, such as an sp3 carbon, at a similar location. This is because rigidly linked atoms tend to give smaller intra-state fluctuations of the reaction coordinate values, making the inter-state differences in reaction coordinate values more prominent. Similarly, uninteresting fluctuations in a reaction coordinate may be suppresed by defining it based on the center of mass of several atoms, instead of on one atom.

\section{Appendix C: atom typing the central double bond}

According to the atom typing rules of GAFF [43], carbon 14 could logically be assigned atom type cc, an "inner sp2 carbon in conjugated ring systems", or atom type ce, an "inner sp2 carbon in conjugated 
chain systems". The parameter assignment program used in this study, Antechamber, would assign cc to all motors considered here. This may be appropriate for Motor $\mathrm{O}$, with its planar stator, but the ring to which carbon 14 belongs is not conjugated for motors $\mathrm{S}$ and $\mathrm{N}$, so type ce seems more appropriate for these two molecules. Indeed, for Motor S and Motor N, using type ce yielded a slightly better value of the objective function in the force field optimization procedures discussed above. In addition, the THI rate constants we computed using ce were generally better than those computed with type cc. Therefore we used ce for motors S and N, with their non-planar stators, and cc for Motor $\mathrm{O}$, with its planar stator.

\section{References}

[1] Kassem S, et al. (2017) Artificial molecular motors. Chem. Soc. Rev. 46(9):2592-2621.

[2] Kolomeisky AB (2013) Motor proteins and molecular motors: how to operate machines at the nanoscale. Journal of Physics: Condensed Matter 25(46):463101.

[3] Slochower DR, Gilson MK (2018) Motor-like Properties of Nonmotor Enzymes. Biophysical Journal 114(9):2174-2179.

[4] Baroncini M, Silvi S, Credi A (2019) Photo- and Redox-Driven Artificial Molecular Motors. Chemical Reviews p. acs.chemrev.9b00291.

[5] Erbas-Cakmak S, Leigh DA, McTernan CT, Nussbaumer AL (2015) Artificial Molecular Machines. Chemical Reviews 115(18):10081-10206.

[6] Feringa BL (2007) The Art of Building Small: From Molecular Switches to Molecular Motors. The Journal of Organic Chemistry 72(18):6635-6652.

[7] Feringa BL (2017) The Art of Building Small: From Molecular Switches to Motors (Nobel Lecture). Angewandte Chemie International Edition 56(37):11060-11078.

[8] García-López V, Liu D, Tour JM (2020) Light-Activated Organic Molecular Motors and Their Applications. Chemical Reviews 120(1):79-124.

[9] Chen J, Kistemaker JCM, Robertus J, Feringa BL (2014) Molecular Stirrers in Action. Journal of the American Chemical Society 136(42):14924-14932.

[10] Eelkema R, et al. (2006) Molecular machines: Nanomotor rotates microscale objects. Nature 440(7081):163-163.

[11] Kudernac T, et al. (2011) Electrically driven directional motion of a four-wheeled molecule on a metal surface. Nature 479(7372):208-211.

[12] Kulago AA, et al. (2010) Ultrafast Light-Driven Nanomotors Based on an Acridane Stator. The Journal of Organic Chemistry 75(3):666-679.

[13] Cnossen A, Kistemaker JCM, Kojima T, Feringa BL (2014) Structural Dynamics of Overcrowded Alkene-Based Molecular Motors during Thermal Isomerization. The Journal of Organic Chemistry 79(3):927-935.

[14] Oruganti B, Fang C, Durbeej B (2015) Computational design of faster rotating second-generation light-driven molecular motors by control of steric effects. Phys. Chem. Chem. Phys. 17(33):2174021751.

[15] Oruganti B, Durbeej B (2016) On the possibility to accelerate the thermal isomerizations of overcrowded alkene-based rotary molecular motors with electron-donating or electron-withdrawing substituents. Journal of Molecular Modeling 22(9):219. 
[16] Klok M, et al. (2008) MHz Unidirectional Rotation of Molecular Rotary Motors. Journal of the American Chemical Society 130(32):10484-10485.

[17] Astumian RD (2016) Optical vs. chemical driving for molecular machines. Faraday Discuss. 195:583-597.

[18] Geertsema EM, van der Molen SJ, Martens M, Feringa BL (2009) Optimizing rotary processes in synthetic molecular motors. Proceedings of the National Academy of Sciences 106(40):1691916924.

[19] Oruganti B, Wang J, Durbeej B (2018) Quantum chemical design of rotary molecular motors. International Journal of Quantum Chemistry 118(1):n/a-n/a.

[20] Liu F, Morokuma K (2012) Computational Study on the Working Mechanism of a Stilbene LightDriven Molecular Rotary Motor: Sloped Minimal Energy Path and Unidirectional Nonadiabatic Photoisomerization. Journal of the American Chemical Society 134(10):4864-4876.

[21] Kazaryan A, et al. (2010) Understanding the Dynamics Behind the Photoisomerization of a LightDriven Fluorene Molecular Rotary Motor. The Journal of Physical Chemistry A 114(15):50585067.

[22] Kazaryan A, Lan Z, Schäfer LV, Thiel W, Filatov M (2011) Surface Hopping Excited-State Dynamics Study of the Photoisomerization of a Light-Driven Fluorene Molecular Rotary Motor. Journal of Chemical Theory and Computation 7(7):2189-2199.

[23] Nikiforov A, Gamez JA, Thiel W, Filatov M (2016) Computational Design of a Family of LightDriven Rotary Molecular Motors with Improved Quantum Efficiency. The Journal of Physical Chemistry Letters 7(1):105-110.

[24] Pang X, et al. (2017) Watching the Dark State in Ultrafast Nonadiabatic Photoisomerization Process of a Light-Driven Molecular Rotary Motor. The Journal of Physical Chemistry A 121(6):12401249.

[25] Vachon J, et al. (2014) An ultrafast surface-bound photo-active molecular motor. Photochem. Photobiol. Sci. 13(2):241-246.

[26] Tuna D, Lu Y, Koslowski A, Thiel W (2016) Semiempirical Quantum-Chemical OrthogonalizationCorrected Methods: Benchmarks of Electronically Excited States. Journal of Chemical Theory and Computation 12(9):4400-4422.

[27] Thiel W (year?) MNDO99 program.

[28] Tully JC (1990) Molecular dynamics with electronic transitions. The Journal of Chemical Physics 93(2):1061-1071. Publisher: American Institute of Physics.

[29] Braun E, et al. (2018) Best Practices for Foundations in Molecular Simulations [Article v1.0]. Living Journal of Computational Molecular Science 1(1):5957.

[30] Martínez L, Andrade R, Birgin EG, Martínez JM (2009) PACKMOL: a package for building initial configurations for molecular dynamics simulations. Journal of computational chemistry 30(13):2157-2164. ISBN: 0192-8651 Publisher: Wiley Online Library.

[31] Wehmeyer C, et al. (2019) Introduction to Markov state modeling with the PyEMMA software [Article v1.0]. Living Journal of Computational Molecular Science 1(1):5965.

[32] Mostofian B, Zuckerman DM (2019) Statistical Uncertainty Analysis for Small-Sample, High LogVariance Data: Cautions for Bootstrapping and Bayesian Bootstrapping. Journal of Chemical Theory and Computation p. acs.jctc.9b00015. 
[33] Zwier MC, et al. (2015) WESTPA: An Interoperable, Highly Scalable Software Package for Weighted Ensemble Simulation and Analysis. Journal of Chemical Theory and Computation 11(2):800-809.

[34] Kästner J (2011) Umbrella sampling: Umbrella sampling. Wiley Interdisciplinary Reviews: Computational Molecular Science 1(6):932-942.

[35] Bonomi M, et al. (2019) Promoting transparency and reproducibility in enhanced molecular simulations. Nature methods 16(8):670-673. ISBN: 1548-7091 Publisher: Nature Publishing Group.

[36] Tribello GA, Bonomi M, Branduardi D, Camilloni C, Bussi G (2014) PLUMED 2: New feathers for an old bird. Computer Physics Communications 185(2):604-613. ISBN: 0010-4655 Publisher: Elsevier.

[37] Grossfield A (year?) WHAM: the weighted histogram analysis method, version 2.0.10.

[38] Kumar S, Rosenberg JM, Bouzida D, Swendsen RH, Kollman PA (1992) THE weighted histogram analysis method for free-energy calculations on biomolecules. I. The method. Journal of Computational Chemistry 13(8):1011-1021. _eprint: https://onlinelibrary.wiley.com/doi/pdf/10.1002/jcc.540130812.

[39] ezá J, Bím D, Gutten O, Rulíek L (2018) Toward Accurate Conformational Energies of Smaller Peptides and Medium-Sized Macrocycles: MPCONF196 Benchmark Energy Data Set. Journal of Chemical Theory and Computation 14(3):1254-1266.

[40] Qiu Y, et al. (2020) Driving torsion scans with wavefront propagation. The Journal of Chemical Physics 152(24):244116.

[41] Parrish RM, et al. (2017) Psi4 1.1: An Open-Source Electronic Structure Program Emphasizing Automation, Advanced Libraries, and Interoperability. Journal of Chemical Theory and Computation 13(7):3185-3197. Publisher: American Chemical Society.

[42] Wang LP, Martinez TJ, Pande VS (2014) Building Force Fields: An Automatic, Systematic, and Reproducible Approach. The Journal of Physical Chemistry Letters 5(11):1885-1891.

[43] Wang J, Wolf RM, Caldwell JW, Kollman PA, Case DA (2004) Development and testing of a general amber force field. Journal of Computational Chemistry 25(9):1157-1174.

[44] He X, Man VH, Yang W, Lee TS, Wang J (2020) A fast and high-quality charge model for the next generation general AMBER force field. The Journal of Chemical Physics 153(11):114502.

[45] Case DA, et al. (year?) AMBER 2018; 2018. University of California, San Francisco.

[46] Jakalian A, Bush BL, Jack DB, Bayly CI (2000) Fast, efficient generation of highquality atomic charges. AM1BCC model: I. Method. Journal of computational chemistry 21(2):132-146. ISBN: 0192-8651 Publisher: Wiley Online Library.

[47] Product C (2012) CRC Handbook of Chemistry and Physics. 92. (Taylor and Francis Group).

[48] Iwata K, Hamaguchi Ho (1997) Microscopic Mechanism of Solute Solvent Energy Dissipation Probed by Picosecond Time Resolved Raman Spectroscopy. The Journal of Physical Chemistry A 101(4):632-637.

[49] Medina J, Avilés F, Tapia A (2015) The bond force constants of graphene and benzene calculated by density functional theory. Molecular Physics 113(11):1297-1305. Publisher: Taylor \& Francis _eprint: https://doi.org/10.1080/00268976.2014.986241.

[50] Jensen F (2017) Introduction to computational chemistry. (John Wiley \& Sons, Chichester, UK ; Hoboken, NJ), Third edition edition. 
[51] Engler EM, Andose JD, Schleyer PVR (1973) Critical evaluation of molecular mechanics. Journal of the American Chemical Society 95(24):8005-8025.

[52] Klok M, Browne WR, Feringa BL (2009) Kinetic analysis of the rotation rate of light-driven unidirectional molecular motors. Physical Chemistry Chemical Physics 11(40):9124.

[53] Klok, and Ben L. Feringa, Jones RAL (2009) The influence of viscosity on the functioning of molecular motors. Faraday Discussions 143:9.

[54] Loncharich RJ, Brooks BR, Pastor RW (1992) Langevin dynamics of peptides: The frictional dependence of isomerization rates of N-acetylalanyl-N-methylamide. Biopolymers 32(5):523-535.

[55] Alberty RA, Goldberg RN (1992) Standard thermodynamic formation properties for the adenosine 5'-triphosphate series. Biochemistry 31(43):10610-10615. ISBN: 0006-2960 Publisher: ACS Publications.

[56] Kinosita K, Yasuda R, Noji H, Ishiwata S, Yoshida M (1998) F1-ATPase: a rotary motor made of a single molecule. Cell 93(1):21-24. ISBN: 0092-8674 Publisher: Elsevier.

[57] Iino R, Hasegawa R, Tabata KV, Noji H (2009) Mechanism of Inhibition by C-terminal Helices of the Subunit of Escherichia coli F oF1-ATP Synthase. Journal of Biological Chemistry 284(26):17457-17464.

[58] Riedel C, et al. (2014) The heat released during catalytic turnover enhances the diffusion of an enzyme. Nature 517(7533):227-230.

[59] Curchod BFE, Martínez TJ (2018) Ab Initio Nonadiabatic Quantum Molecular Dynamics. Chemical Reviews 118(7):3305-3336.

[60] Kistemaker JCM, et al. (2017) Third-Generation Light-Driven Symmetric Molecular Motors. Journal of the American Chemical Society 139(28):9650-9661.

[61] Zuckerman DM (2019) Physical Lens on the Cell.

[62] Cremer D, Pople JA (1975) General definition of ring puckering coordinates. Journal of the American Chemical Society 97(6):1354-1358. 


\title{
Electronic Supplementary Information for PCCP Article: Mechanistic Analysis of Light-Driven Overcrowded Alkene-Based Molecular Motors by Multiscale Molecular Simulations
}

\author{
Mudong Feng and Michael K. Gilson
}

December 29, 2020

\begin{abstract}
Department of Chemistry and Biochemistry, and Skaggs School of Pharmacy and Pharmaceutical Sciences, UC San Diego, La Jolla, CA 92093
\end{abstract}

This document provides additional data and details settings of the calculations mentioned in the main text. To reproduce or adapt these calculations, see Github repository github.com/fengmudong/motorpaper, where all the input files and associated instructions can be found.

\section{Additional Figures}
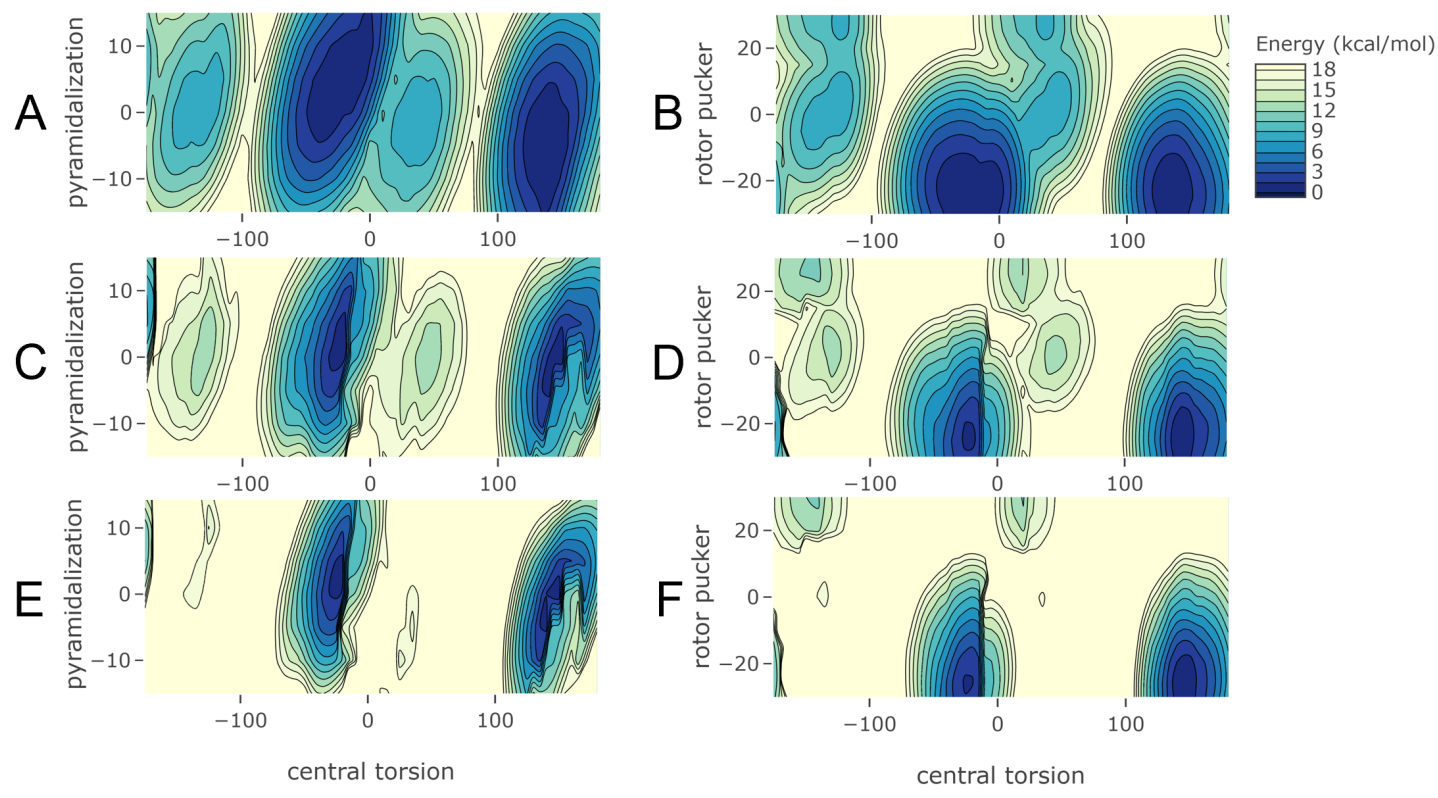

Figure S1: Potential energy surfaces of Motor N. A,B: quantum mechanical (QM) energy. C,D: energy from forcefield after fitting to QM. E,F: energy from GAFF2 forcefield, without any fitting to QM. The scanned coordinates are the central torsion angle, 18-15-14-10; the pyramidalization improper dihedral, 14-1-13-15; and the rotor pucker dihedral, 27-15-16-25. The force field energy for each grid point was evaluated at the corresponding QM conformation without further relaxation. For this larger molecule, the QM calculations used a smaller basis set, 3-21G. 


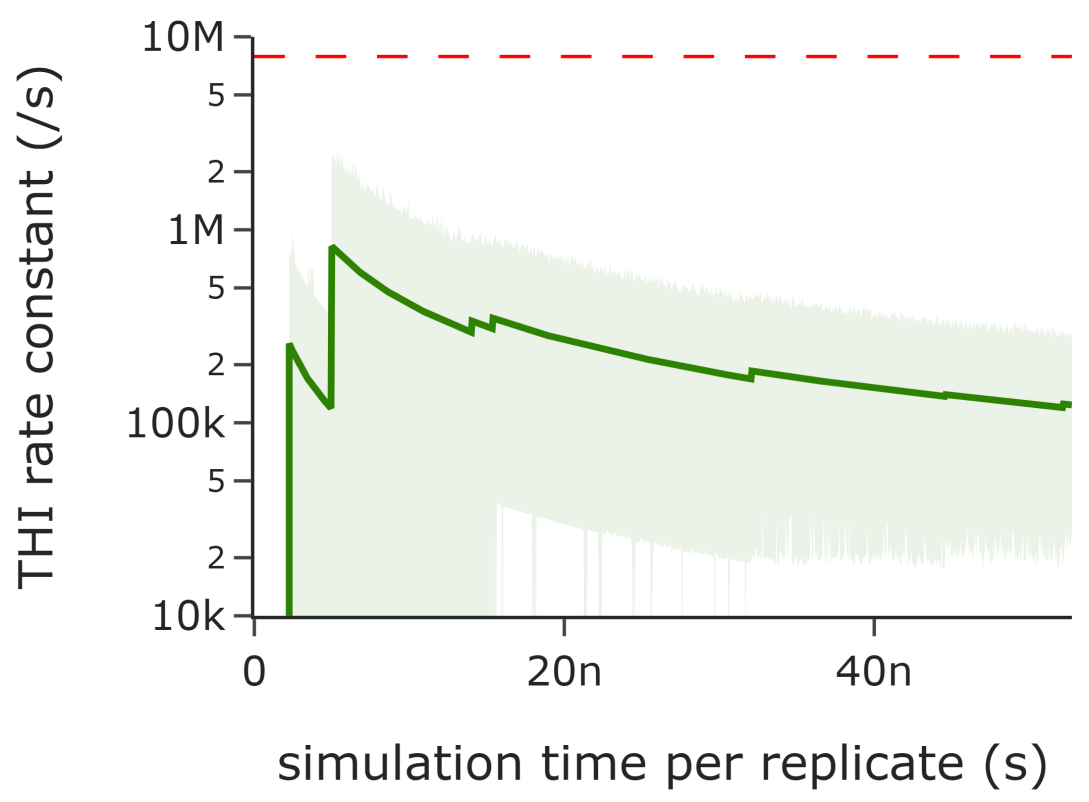

Figure S2: THI rate constant computed with WESTPA of Motor S under $3.4 \mathrm{kcal} \mathrm{mol}^{-1}$ load, as a function of simulation time per replicate, with shading to indicte $95 \%$ bootstrapping confidence interval. Dashed red line indicates experimental rate constant of the non-loaded motor at $300 \mathrm{~K}, 7.9 \times 10^{6} \mathrm{~s}^{-1}$, read from Arrhenius plots of Klok et al.[1].

\section{Cartesian Coordinates of Selected Structures}

Files MS1.pdb, MS2.pdb, MS3.pdb, and MS4.pdb each contains 10 sample conformations of Motor S metastable substates MS1, MS2, MS3, MS4, respectively. File stable.pdb contains a DFT optimized structure of the stable form of Motor S. For more technical purposes or for reproducing calculations in this study, more Cartesian structures in various formats can be found in the Github repository.

\section{Trajectory Videos}

Four trajectories videos are mentioned in the main text to animate the motor rotation process, and are available online. To make thermal fluctuations less distracting, the trajectory videos were smoothed in VMD [2] by averaging over a 20 frame moving window. We did not observe any noteworthy solvent motion, so solvent molecules were stripped out. To facilitate visualization, center-of-mass translation and rotation were removed and the stator atoms were aligned across snapshots to make the stator appear steady.

\section{Settings Related to Surface-hopping simulations}

To describe the photoexcitation leading to central double bond rotation, we chose a minimal active space consisting of the two $\pi$ bonded orbitals on the two central carbon atoms. Electron correlation was computed to the CISD level. During MD, the active space was tracked by an overlap criterion so that the molecular orbitals retain their character, to a threshold of at least 70 percent overlap with previous active space. The time step of nuclear motion was $0.5 \mathrm{fs}$ for ground state sampling simulations, or $0.1 \mathrm{fs}$ for most surface-hopping simulations. For motor N, which has more atoms and slower calculations, we increased the surface-hopping timestep to $0.2 \mathrm{fs}$. We found the $0.2 \mathrm{fs}$ simulation results of Motor $\mathrm{N}$ to be similar to $0.1 \mathrm{fs}$ results of Motor $\mathrm{N}$. 


\section{Settings Related to Plain Classical MD Simulations}

The short-ranged PME cutoff was $8 \AA$, the time step was $2 \mathrm{fs}$, the SHAKE algorithm was used to constrain bonds involving hydrogen, and a snapshot was written to disk every 400 steps. The Monte Carlo barostat was used, with a compressibility of comp=103 (in unit $1 \times 10^{-6} \mathrm{bar}^{-1}$ ), based on measured compressibility of dichloromethane.

\section{Settings Related to WESTPA Simulations}

The weighted ensemble MD simulations used the same parameters used in above plain MD simulations. We found that whether or not SHAKE was used does not affect the computed rate constant. After each WESTPA iteration of 50ps, replicates were examined for cloning or merging, for the target of having 20 replicates in each bin. For Motor S, the bin boundaries, in degrees, were -180, -80, -70, -60, $-50,-40,-30,-20,-10,0,10,20,30,40,60,80,100,120,140,160,180$ for the first progress coordinate, and $0,20,100,120,140,160,180,200,220,240,260,280,360$ for the second. For Motor N, we used slightly different bin boundaries in the first coordinate: $-180,-60,-50,-40,-30,-20,-10,0,10,20,30$, 40, 60, 80, 100, 120, 140, 180 .

\section{Settings related to MSM}

The high dimensional trajectories of atomic positions were first condensed to time series of all the dihedrals in the solute motor's topology file, each associated with a forcefield term. The time series were calculated at intervals of $0.8 \mathrm{ps}$. This interval was also used as the lag time, i.e., the time resolution of the MSM. We also tested lag times up to $80 \mathrm{ps}$, and found that longer lag times did not significantly change the computed rate constants. Then the number of dimensions were further reduced to 8 for Motor S, and 19 for Motor N, by using time-lagged independent component analysis (TICA) on the time series. The number of dimensions was chosed as the minimal number that still preserves high VAMP2 score. Next, the continuous time series were discretized to 64 conformational clusters using k-means clustering. Then the clusters were classified by Perron cluster analysis (PCCA) [3] into four metastable substates. We used sharp classification instead of fuzzy probabilistic classification, so that pairs of substates that do not interconvert directly receive transition counts of exactly zero rather than small numbers. Then the MSM, as a 4x4 transition matrix, was computed from the time series of interconversion among these four substates. The Chapman-Kolmogorov test [3] was conducted which validates the MSM. Our workflow is available in a more detailed and easily reproducible format, as Jupyter notebooks in our Github repository.

\section{Settings Related to Umbrella Sampling Simulations}

MD simulation parameters are the same as in plain MD. All simulation windows start with the same AMBER restart file used in plain MD; in the restart file, the motor is at metastable form and the system has been equilibrated for plain MD. The spring constant of the window restraints were determined by trials; we found that a too large spring constant may crash the simulation, while a too small spring constant may fail to pull the reaction coordinate near the window center. The first 100 frames of each window were excluded from analysis to eliminate pulling transients.

\section{References}

[1] M. Klok, N. Boyle, M. T. Pryce, A. Meetsma, W. R. Browne, and B. L. Feringa, "MHz Unidirectional Rotation of Molecular Rotary Motors," Journal of the American Chemical Society, vol. 130, pp. 10484-10485, Aug. 2008.

[2] W. Humphrey, A. Dalke, and K. Schulten, "VMD Visual Molecular Dynamics," Journal of Molecular Graphics, vol. 14, pp. 33-38, 1996. 
[3] C. Wehmeyer, M. K. Scherer, T. Hempel, B. E. Husic, S. Olsson, and F. Noé, "Introduction to Markov state modeling with the PyEMMA software [Article v1.0]," Living Journal of Computational Molecular Science, vol. 1, no. 1, p. 5965, 2019. 